\title{
Atomic-Scale in Situ Observations of Crystallization and Restructuring Processes in Two-Dimensional $\mathrm{MoS}_{2}$ Films
}

Bernhard C. Bayer, ${ }^{*},+\ddagger$ Reinhard Kaindl, ${ }^{\S}$ Mohammad Reza Ahmadpour Monazam, ${ }^{\dagger}$ Toma Susi, ${ }^{\dagger}$ Jani Kotakoski, ${ }^{\dagger}$ Tushar Gupta, $^{\ddagger}$ Dominik Eder, ${ }^{\ddagger \odot}$ Wolfgang Waldhauser, ${ }^{\S}$ and Jannik C. Meyer ${ }^{\dagger \odot}$

${ }^{\dagger}$ Faculty of Physics, University of Vienna, Boltzmanngasse 5, A-1090 Vienna, Austria

${ }^{\ddagger}$ Institute of Materials Chemistry, Vienna University of Technology (TU Wien), Getreidemarkt 9, A-1060 Vienna, Austria

$\S$ Joanneum Research - Materials, Institute of Surface Technologies and Photonics, Leobner Straße 94, A-8712 Niklasdorf, Austria

Supporting Information

ABSTRACT: We employ atomically resolved and elementspecific scanning transmission electron microscopy (STEM) to visualize in situ and at the atomic scale the crystallization and restructuring processes of two-dimensional (2D) molybdenum disulfide $\left(\mathrm{MoS}_{2}\right)$ films. To this end, we deposit a model heterostructure of thin amorphous $\mathrm{MoS}_{2}$ films onto freestanding graphene membranes used as high-resolution STEM supports. Notably, during STEM imaging the energy input from the scanning electron beam
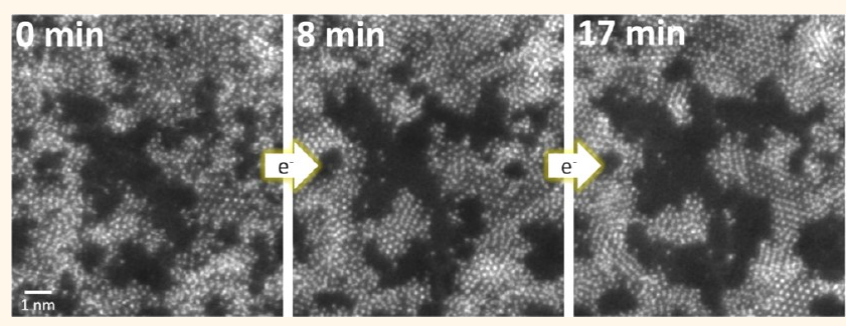

leads to beam-induced crystallization and restructuring of the amorphous $\mathrm{MoS}_{2}$ into crystalline $\mathrm{MoS}_{2}$ domains, thereby emulating widely used elevated temperature $\mathrm{MoS}_{2}$ synthesis and processing conditions. We thereby directly observe nucleation, growth, crystallization, and restructuring events in the evolving $\mathrm{MoS}_{2}$ films in situ and at the atomic scale. Our observations suggest that during $\mathrm{MoS}_{2}$ processing, various $\mathrm{MoS}_{2}$ polymorphs co-evolve in parallel and that these can dynamically transform into each other. We further highlight transitions from in-plane to out-of-plane crystallization of $\mathrm{MoS}_{2}$ layers, give indication of Mo and $S$ diffusion species, and suggest that, in our system and depending on conditions, $\mathrm{MoS}_{2}$ crystallization can be influenced by a weak $\mathrm{MoS}_{2}$ /graphene support epitaxy. Our atomic-scale in situ approach thereby visualizes multiple fundamental processes that underlie the varied $\mathrm{MoS}_{2}$ morphologies observed in previous ex situ growth and processing work. Our work introduces a general approach to in situ visualize at the atomic scale the growth and restructuring mechanisms of $2 \mathrm{D}$ transition-metal dichalcogenides and other 2D materials.

KEYWORDS: $\mathrm{MoS}_{2}$ graphene, aberration-corrected scanning transmission electron microscopy, in situ, physical vapor deposition, crystallization, two-dimensional heterostructures

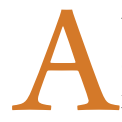

tomically resolved in situ observations of the growth and structural evolution of two-dimensional (2D) materials during realistic processing remain a difficult challenge by (scanning) transmission electron microscopy ((S)TEM). Two factors contribute to this: First, many 2D materials require a solid growth support with a thickness that impedes electron transparency. This often restricts in situ (S)TEM experimentation to cross-sectional sample arrangements ${ }^{1}$ and precludes potentially more informative plan view sample geometries under the electron beam (e-beam). Second, growth of many 2D materials, via for instance, chemical vapor deposition (CVD) or physical vapor deposition (PVD) techniques, requires temperatures and gas pressures that can be challenging to achieve in (environmental) (S)TEM. ${ }^{2-4}$

Addressing both points, we here provide an approach to achieve atomically resolved and element-specific in situ STEM plan view imaging of the crystallization and restructuring processes in $2 \mathrm{D}$ materials, shown here for the important $2 \mathrm{D}$ transition-metal dichalcogenide (TMDC) molybdenum disulfide $\left(\mathrm{MoS}_{2}\right)$. To this end, we fabricate a model heterostructure system by depositing ultrathin amorphous $\operatorname{MoS}_{2}\left(\mathrm{a}-\mathrm{MoS}_{2}\right)$ films on graphene membranes, which act as ideal STEM supports. ${ }^{5}$ When these model samples are imaged in STEM we notably find that the energy input ${ }^{6}$ from the scanning e-beam emulates $\mathrm{MoS}_{2}$ processing at elevated temperature (such as occurring in CVD, PVD, or general annealing treatments), leading to e-beam-induced crystallization and restructuring of the $\mathrm{MoS}_{2}$. By this approach of using the STEM e-beam to both probe and modify the material, we directly follow how a-MoS

Received: June 29, 2018

Accepted: August 3, 2018

Published: August 3, 2018 


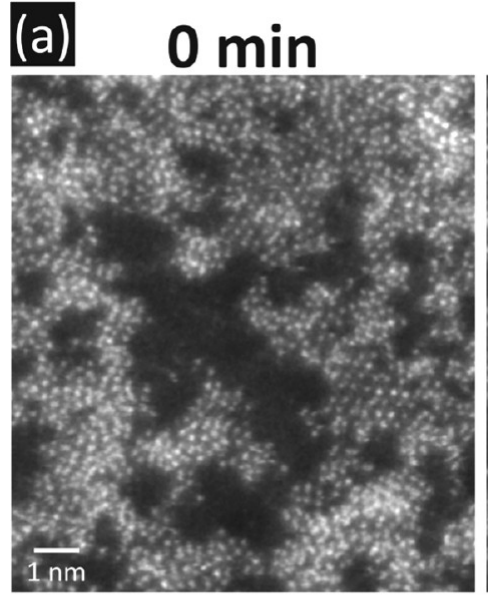

$14 \mathrm{~min}$

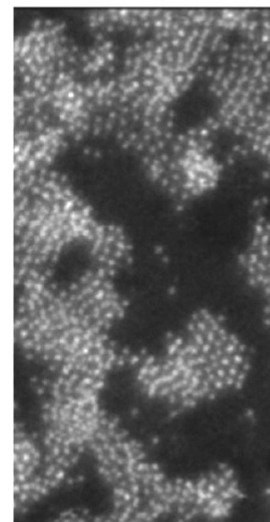

(c)

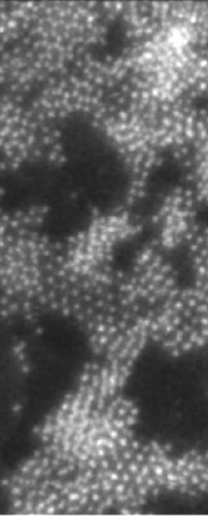

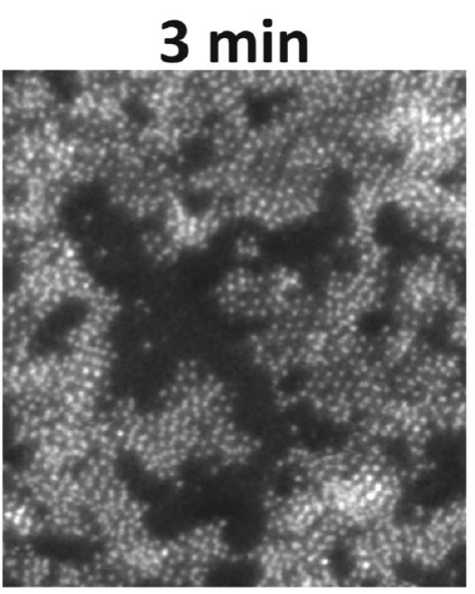

$15 \mathrm{~min}$

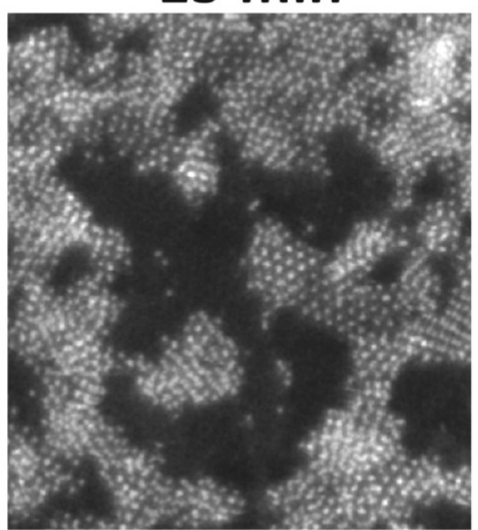

$\mathrm{a}-\mathrm{MoS}_{2}$

\section{graphene}

support

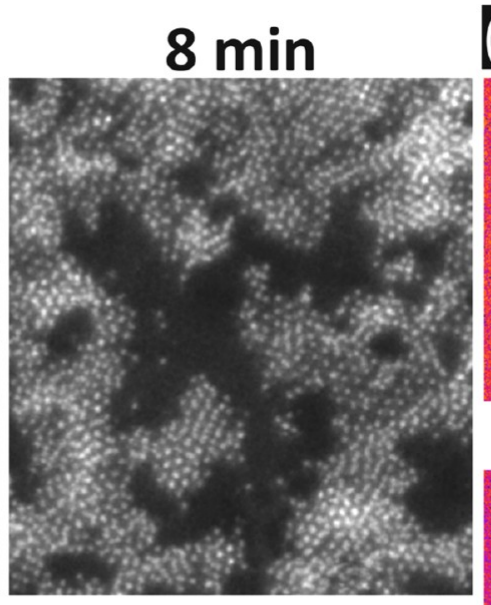

(b) $0 \mathrm{~min}$

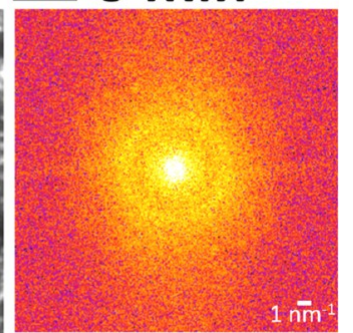

$8 \mathrm{~min}$

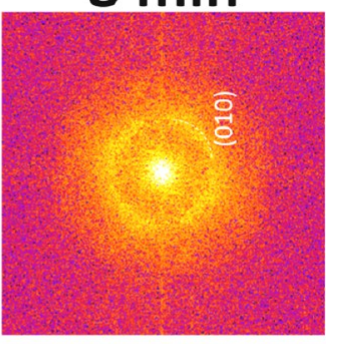

$17 \mathrm{~min}$
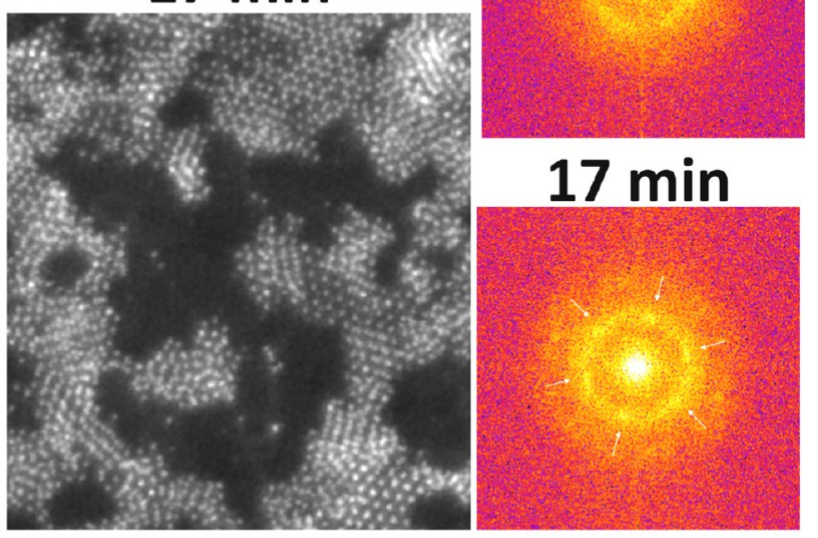

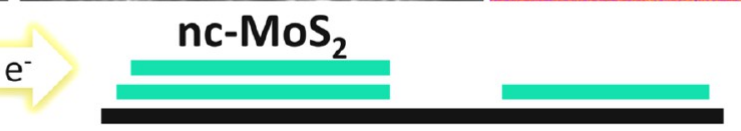

Figure 1. (a) HAADF STEM image series of $\sim 2 \mathrm{~nm} \mathrm{MoS}$ on graphene during continuous e-beam exposure (time stamps indicated) which leads to e-beam-induced crystallization from a- $\mathrm{MoS}_{2}$ to $\mathrm{nc}-\mathrm{MoS}_{2}$ domains. (b) FTs of selected frames in (a) with corresponding time stamps indicated. (c) Schematic illustration summarizing the observations deduced from (a).

films crystallize and restructure to nanocrystalline $\mathrm{MoS}_{2}$ (nc$\mathrm{MoS}_{2}$ ) domains and thereby explore in situ and at the atomic scale the richness of $\mathrm{MoS}_{2}$ 's structural evolution via multiple polymorphs.

The importance of $\mathrm{MoS}_{2}$ stems from the current interest to use this material as a device active layer in low-dimensional (opto-)electronics ${ }^{7}$ as well as a potent catalyst in (photo)electrochemical energy applications, such as the hydrogen evolution reaction (HER). ${ }^{8-10}$ All of these application fields share the key prerequisite of scalable synthesis of $\mathrm{MoS}_{2}$ with controlled properties. The desired structural characteristics of $\mathrm{MoS}_{2}$ in electronic and catalytic applications vary however drastically: For electronics, semiconducting $\mathrm{MoS}_{2}$ with precisely controlled layer number, large crystals, and a low defect density is desired in order to achieve, for example, high current on/off ratios and high carrier mobilities in field effect transistor (FET) $\mathrm{MoS}_{2}$ devices. ${ }^{7}$ In stark contrast, for (electro)catalytic applications such as HER typically finely nanostructured or even amorphous $\mathrm{MoS}_{2}$ with good electrical conductivity, a large specific surface area and a large number of pronounced defects and edge sites are desired, since these imperfections rather than a highly crystalline basal plane are considered as electrocatalytically active sites. ${ }^{8,11}$
Important in this context, $\mathrm{MoS}_{2}$ occurs in multiple polymorphs: First on the monolayer level, the arrangement of the three covalently bonded atomic sublayers (S-Mo-S) within a $\mathrm{MoS}_{2}$ monolayer can principally show trigonal prismatic (commonly termed " $2 \mathrm{H}$ monolayer", also often referred to as " $1 \mathrm{H}$ monolayer") or octahedral (termed " $1 \mathrm{~T}$ monolayer") symmetries. ${ }^{12-15}$ Importantly, the more commonly found $2 \mathrm{H}$ monolayers are semiconducting, while the comparatively metastable $1 \mathrm{~T}$ monolayers are metallic, implying a key influence of $\mathrm{MoS}_{2}$ monolayer symmetry on the material's application profile. Second, when individual monolayers of a given symmetry type are stacked upon each other by van der Waals interactions, multiple stacking arrangements are possible, which in turn impact on optoelectronic properties. For instance, for the $2 \mathrm{H}$ monolayer type, several stacking arrangements are possible, where the most commonly occurring equilibrium types are $2 \mathrm{H}\left(\mathrm{AA}^{\prime}\right.$ stacking) and $3 \mathrm{R}$ (ABC stacking). ${ }^{16-20}$ Importantly beyond the equilibrium $2 \mathrm{H}$ and $3 \mathrm{R}$ stacking, also more complex nonequilibrium stacking sequences including homonuclear stacking (e.g., AA) have been reported. ${ }^{17}$ With increasing layer number, the possible complexity of these layer arrangements generally increases, since different stacking types can also co-exist within 
multilayers, ${ }^{17,19,21,22}$ let alone given the further additionally complex possibility of different monolayer types $(2 \mathrm{H} / 1 \mathrm{~T})$ stacking onto each other. This polymorphism of $\mathrm{MoS}_{2}$ in both monolayer type and multilayer stacking opens a complex parameter space of possible layered $\mathrm{MoS}_{2}$ structures which require control in any synthesis route.

The key methods to realize $\mathrm{MoS}_{2}$ layers are CVD (including solid metal/vapor sulfurization methods), ${ }^{23-28}$ PVD (e.g., sputter deposition or evaporation), ${ }^{29-34}$ and wet chemical synthesis. ${ }^{10,35}$ Important in this context is that, unlike other key $2 \mathrm{D}$ materials (such as graphene ${ }^{1}$ or hexagonal boron nitride $\left.{ }^{36}\right), \mathrm{MoS}_{2}$ does not require a metallic process catalyst to grow and crystallize. Also $\mathrm{MoS}_{2}$ growth can be achieved already at comparatively low temperatures $\left(\sim 400{ }^{\circ} \mathrm{C}\right)$. Based on this comparatively facile crystallization of $\mathrm{MoS}_{2}$, CVD-type synthesis is most promising for electronic-grade $\mathrm{MoS}_{2}$, while PVD and wet chemical synthesis offer a high degree of control over nanostructured electrocatalytically active $\mathrm{MoS}_{2}$. While the various $\mathrm{MoS}_{2}$ synthesis techniques comprise completely different formation environments, precursors, constituent species fluxes, and significantly different growth kinetics, all techniques nevertheless routinely employ elevated temperature treatments $\left(\sim 400{ }^{\circ} \mathrm{C}\right)$ at some stage during growth or postprocessing in order to stabilize a certain $\mathrm{MoS}_{2}$ structure. Therefore, in all synthesis routes of $\mathrm{MoS}_{2}$ the structural mechanisms proceeding at elevated temperatures such as nucleation, sustained growth, crystallization, and restructuring are of fundamental importance. Yet, at present little work has been done to elucidate these mechanisms. ${ }^{11,37-40}$ In particular, in situ observations of growth, crystallization, and restructuring processes in $\mathrm{MoS}_{2}$ and other TMDCs at the atomic scale are critically missing. This results in a limited understanding of the fundamental mechanisms underlying synthesis and processing, thereby hindering rational synthesis and postgrowth process development for $\mathrm{MoS}_{2}$.

Our here presented approach for atomically resolved in situ STEM imaging of $\mathrm{MoS}_{2}$ crystallization and restructuring therefore contributes to such much needed understanding by directly identifying various mechanistic growth and restructuring steps: In particular we observe, depending on the initial thickness of a- $\mathrm{MoS}_{2}$ deposited, in-plane crystallization toward few-layer nc- $\mathrm{MoS}_{2}$ with layers parallel to the support for thin a$\mathrm{MoS}_{2}$ films, while comparatively thicker a- $\mathrm{MoS}_{2}$ films evolve into a two-segment nc- $\mathrm{MoS}_{2}$ film morphology with interfacial in-plane $\mathrm{MoS}_{2}$ layer crystallization parallel to the support and with perpendicular $\mathrm{MoS}_{2}$ layering farther away from the support. Our data reveal that during this crystallization and restructuring various $\mathrm{MoS}_{2}$ polymorphs co-evolve in parallel. Importantly, we find that these polymorphs dynamically transform into each other during processing, driven via diffusion of Mo and S species and, depending on conditions, influenced by a weak $\mathrm{MoS}_{2}$ /graphene heterostructure support epitaxy. Contextualizing these in situ observations with recent ex situ $\mathrm{MoS}_{2}$ growth and processing literature, our work visualizes in situ and at the atomic scale the multiple fundamental structural processes occurring in parallel under widely used ex situ $\mathrm{MoS}_{2}$ processing conditions. Notably, our insights are based on a model system and a STEM environment that is readily extendable to in situ studies of other TMDCs and 2D materials.

\section{RESULTS AND DISCUSSION}

We fabricate a-MoS 2 samples for high-resolution STEM by sputter deposition of ultrathin PVD $\mathrm{MoS}_{2}$ films directly onto free-standing monolayer CVD graphene membranes. The graphene membranes, which act as a ultrathin and light support for STEM, ${ }^{5}$ were suspended across the holes of a holey carbon support foil of a TEM grid by a polymer-free transfer process which ensures an as clean as possible $\mathrm{MoS}_{2}$-graphene interface. ${ }^{36,41-43}$ During PVD of $\mathrm{MoS}_{2}$ onto the graphenecovered TEM grids, the samples were not intentionally heated leading to deposition of a-MoS${ }_{2}{ }^{34} \mathrm{MoS}_{2}$ film with nominal thicknesses ranging from $\sim 2 \mathrm{~nm}$ to $\sim 10 \mathrm{~nm}$ were deposited. For further details on experimental methods, see the Methods section.

Figure 1a shows a high-angle annular dark-field (HAADF) STEM image series (60 kV electron acceleration voltage) of a nominally $\sim 2 \mathrm{~nm}$-thick $\mathrm{MoS}_{2}$ film on a graphene monolayer during its structural evolution as a function of continuous ebeam scanning time (time stamps indicated in Figure 1; sample was not intentionally heated during STEM imaging). As apparent from the initial image at $0 \mathrm{~min}$, the $\sim 2 \mathrm{~nm} \mathrm{MoS}$ in its as-deposited state does not homogeneously cover the graphene support but shows a morphology of interconnected islands (bright regions in Figure 1) with bare graphene areas in between (dark regions in Figure 1). The STEM image at $0 \mathrm{~min}$ also gives the visual impression of an amorphous structure in the $\mathrm{MoS}_{2}$ deposit. This is corroborated by the Fourier transform (FT) data in Figure $1 \mathrm{~b}$ for $0 \mathrm{~min}$, which only shows a broad halo indicative of amorphicity. Upon continued scanning of the e-beam over the field of view of Figure 1a, the appearance of the $\mathrm{MoS}_{2}$ islands gradually changes: The visual impression suggests gradual island restructuring resulting in (i) crystallization of the amorphous $\mathrm{MoS}_{2}$ islands toward nc-MoS with the $\mathrm{MoS}_{2}$ layers parallel to the graphene support as well as (ii) slight "dewetting" of the $\mathrm{MoS}_{2}$ from its support. Corroborating a- $\mathrm{MoS}_{2}$ crystallization, after $8 \mathrm{~min}$ e-beam exposure not only the visual appearance in the STEM data (Figure 1a) but also the FT data in Figure 1b suggest some ebeam-induced crystallization of the $\mathrm{MoS}_{2}$ as a more pronounced ring in the FT pattern has emerged. The emerging ring corresponds well to the $2 \mathrm{H} \mathrm{MoS}_{2}(010)$ reflection $(\sim 0.26$ $\mathrm{nm})$, consistent with $2 \mathrm{H} \mathrm{MoS}$ crystallization with layers parallel to the support. After $17 \mathrm{~min}$ of continuous e-beam scanning, the FT in Figure $1 \mathrm{~b}$ has even developed signs of one discrete hexagonal spot pattern (indicated by white arrows). This suggests an emerging dominant crystalline $2 \mathrm{H} \mathrm{MoS}_{2}$ layer orientation across the entire field of view in Figure 1a (17 min). Interestingly, we find that the e-beam-induced crystallization is a phenomenon highly localized to the ebeam in STEM with a sharp boundary between exposed crystallized and nonexposed amorphous material (Figure 2). We note that such a good spatial definition of the beam-driven crystallization implies that e-beam exposure could potentially be used to spatially selectively transform a-MoS 2 to $\mathrm{nc}-\mathrm{MoS}_{2}$ in a fabrication scenario. ${ }^{6}$ Given the lower chemical stability of a$\mathrm{MoS}_{2}$ compared to nc-MoS${ }_{2}{ }^{34}$ this may be useful for direct resist-free patterning of crystalline $\mathrm{MoS}_{2}$ devices where nonexposed a- $\mathrm{MoS}_{2}$ could be chemically etched away with the more stable crystalline $\mathrm{MoS}_{2}$ remaining.

To complement our STEM measurements, additional timeresolved bright-field (BF) transmission electron microscopy (TEM) and selected area electron diffraction (SAED) 


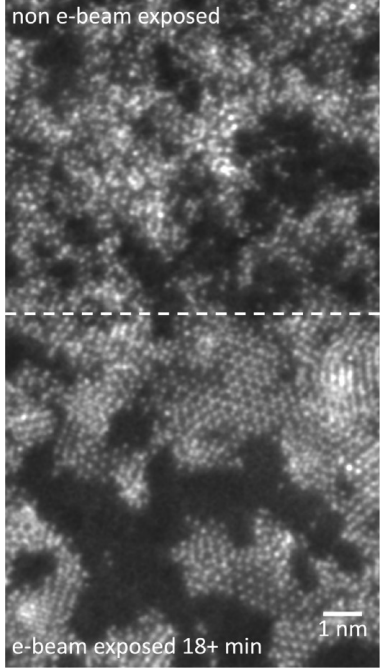

Figure 2. HAADF STEM image of the $\sim 2 \mathrm{~nm} \mathrm{MoS}$ on graphene corresponding to Figure 1a. In the lower half of the image the $\mathrm{MoS}_{2}$ has been exposed to $18+\mathrm{min}$ of continuous e-beam scanning, leading to crystallization of the initial a-MoS $\mathrm{M}_{2}$ to nc$\mathrm{MoS}_{2}$ domains. In contrast, in the upper half of the image the $\mathrm{MoS}_{2}$ has not been previously e-beam exposed, therefore remaining in its as-deposited a- $\mathrm{MoS}_{2}$ state. The sharp boundary between the a-MoS ${ }_{2}$ and the nc-MoS $($ dotted white line) indicates that the e-beam-induced crystallization is a phenomenon highly localized to the area exposed to the e-beam with nm-scale resolution.

measurements at electron acceleration voltages from $60 \mathrm{kV}$ to $200 \mathrm{kV}$ are presented in Supporting Information Figures S1S3. Figure $S 1(80 \mathrm{kV})$ corroborates at a wider field of view in the TEM (up to $900 \mathrm{~nm}$ ) the e-beam-induced restructuring and crystallization of our $\sim 2 \mathrm{~nm}$ a- $\mathrm{MoS}_{2}$ films to $\mathrm{nc}-\mathrm{MoS}_{2}$ with $2 \mathrm{H} \mathrm{MoS}$ layers parallel to the graphene support, consistent with our STEM data. Figure S2 $(60 \mathrm{kV}$, same electron acceleration voltage as used in STEM) shows similar e-beaminduced crystallization at $60 \mathrm{kV}$ and reveals via time-dependent energy dispersive X-ray spectroscopy (EDX) measurements that the $S /$ Mo ratio in the films during their e-beam-induced transition from a- $\mathrm{MoS}_{2}$ to nc- $\mathrm{MoS}_{2}$ only slightly drops from S/ $\mathrm{Mo}_{0 \text { min }}=2.1 \pm 0.03$ to $\mathrm{S} / \mathrm{Mo}_{20 \text { min }}=2.0 \pm 0.03$. This suggests that the loss of $\mathrm{S}$ via e-beam-induced sputtering processes in our $\mathrm{MoS}_{2}$-graphene heterostructures in particular at $60 \mathrm{kV}$ can remain limited, consistent with previous literature. ${ }^{44,45}$ Figure
S3 $(80 \mathrm{kV}$ vs $200 \mathrm{kV})$ finally confirms that a-MoS crystallization is also observed for $200 \mathrm{kV}$ electron acceleration, whereby we find that the rate of a- $\mathrm{MoS}_{2}$ crystallization for 80 $\mathrm{kV}$ and $200 \mathrm{kV}$ appears roughly similar, while in contrast the degradation rate of the graphene support is much more pronounced at $200 \mathrm{kV}$ due to much increased electron knockon damage to the graphene. ${ }^{46}$ Overall and most importantly, our TEM data in Figures $\mathrm{S} 1-\mathrm{S} 3$ confirm that the observed ebeam-induced a- $\mathrm{MoS}_{2}$ crystallization is a generic processes independent of our particular employed microscope type (i.e., STEM or TEM; note that one STEM and two different TEM systems were found to give consistent results, see Methods section) and is working over a wide range of typical (S)TEM electron acceleration voltages and imaging parameters, making our model heterostructures an easily implemented in situ imaging platform.

Figure 1c schematically illustrates our observations of this ebeam-induced crystallization and restructuring of initial a$\mathrm{MoS}_{2}$ clusters to nc-MoS 2 of a few layers thickness with $\mathrm{MoS}_{2}$ planes parallel to its graphene support. Atomic-scale in situ work on $\mathrm{MoS}_{2}$ has to date primarily concentrated on the formation of defects in and amorphization of initially fully crystalline $\mathrm{MoS}_{2}$ monolayers, ${ }^{44,47-50}$ that is, the reverse process of the a- $\mathrm{MoS}_{2}$ crystallization observed here and on phase transitions (e.g., $2 \mathrm{H}$ to $1 \mathrm{~T}$ ) in fully crystalline $\mathrm{MoS}_{2}{ }^{13} \mathrm{In}$ contrast, crystallization of a-MoS 2 , as followed here at the atomic scale, has previously been studied only at comparatively large fields of view, insufficient to discern details on the single atom level, be it in or ex situ from thermal activation $^{11,37-40,51,52}$ or e-beam irradiation..$^{39,52-54}$ In contrast to previous work, our high-resolution STEM data now allow us to discuss atomic-scale details of the crystallization and restructuring processes based on direct in situ information.

To quantify the HAADF STEM intensity data from Figure $1 \mathrm{a}$, we show in Figure $3 \mathrm{a}$ the central region from Figure 1a at higher magnification after $18 \mathrm{~min}$ e-beam exposure. Taking a HAADF intensity line profile (Figure $3 b$ ) along the yellow line indicated in Figure $3 \mathrm{a}$ allows to identify the nature of the atoms in the image based on the element-specific intensity of HAADF data of ultrathin films which has a dependence ${ }^{55}$ on atomic number $Z$ of $Z^{\sim 1.64}$. We thereby identify the thinnest region in Figure $3 \mathrm{a}$ (across which the line profile is drawn) to be a $\mathrm{MoS}_{2}$ monolayer of $2 \mathrm{H}$ monolayer structure ${ }^{12,13}$ (Figure $3 \mathrm{~b}$ inset). Consistently this region displays a 6-fold FT (inset of Figure 3a) with distances of $\sim 0.26 \mathrm{~nm}$ and $\sim 0.15 \mathrm{~nm}$ corresponding to the (010) and (110) reflections of $2 \mathrm{H} \mathrm{MoS}_{2}$,
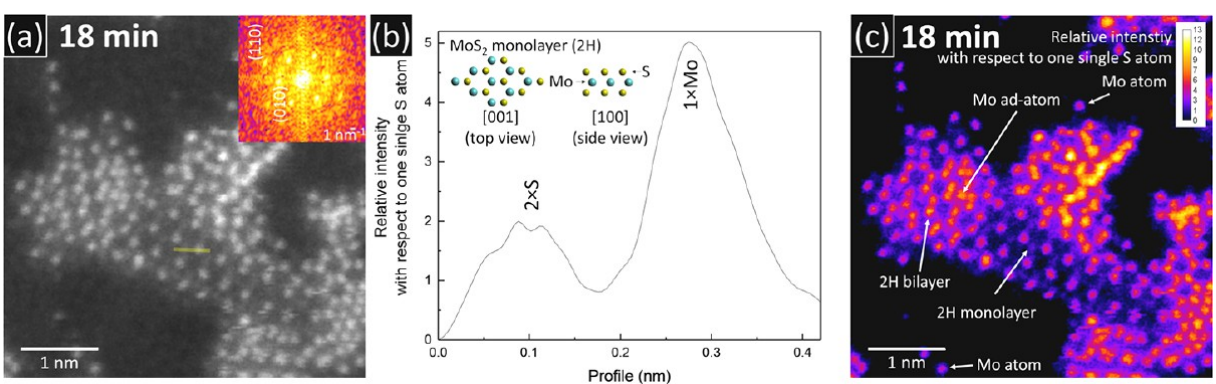

Figure 3. (a) HAADF STEM image of a $\sim 2 \mathrm{~nm} \mathrm{MoS}$ island (zoom-in to the central region of Figure 1) after 18 min continuous e-beam exposure. The inset shows the corresponding FT. (b) Line profile drawn along the yellow line in (a) for which HAADF intensity has been normalized to the intensity of a single $S$ atom. ${ }^{55}$ The identified positions of $S$ and Mo atoms are labeled. The inset shows a schematic topand side-view of a $2 \mathrm{H} \mathrm{MoS}$ monolayer. (c) False colored recalculation of (a) for which HAADF intensity has been normalized to the intensity of a single $S$ atom. 

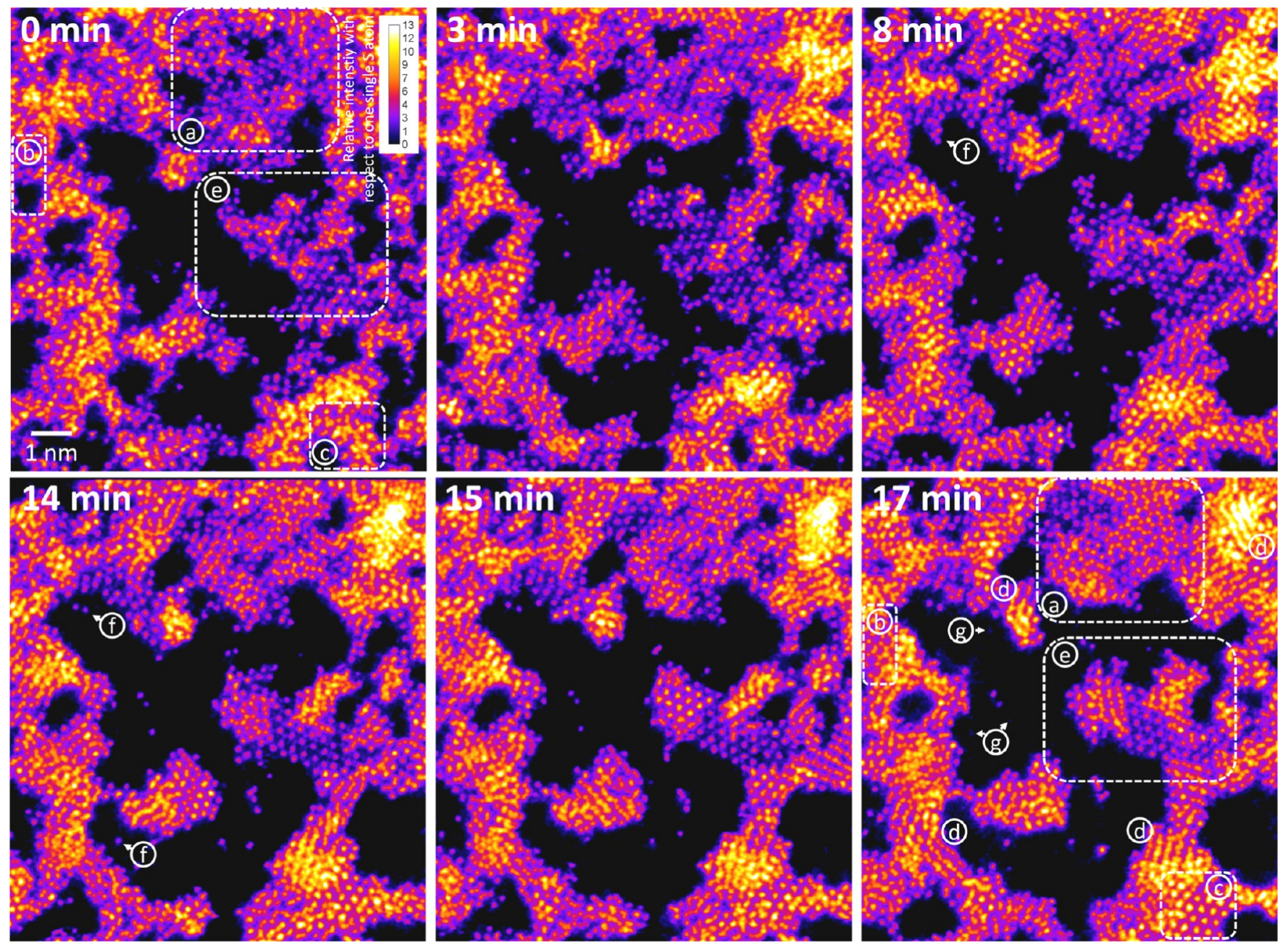

Figure 4. False colored recalculation of the in situ crystallization time series in Figure 1 (time stamps indicated) for which the HAADF intensity has been normalized to the intensity of a single $S$ atom. ${ }^{55}$ The labeled spots (a) to (g) point to salient structural features and evolutions discussed in the main text.
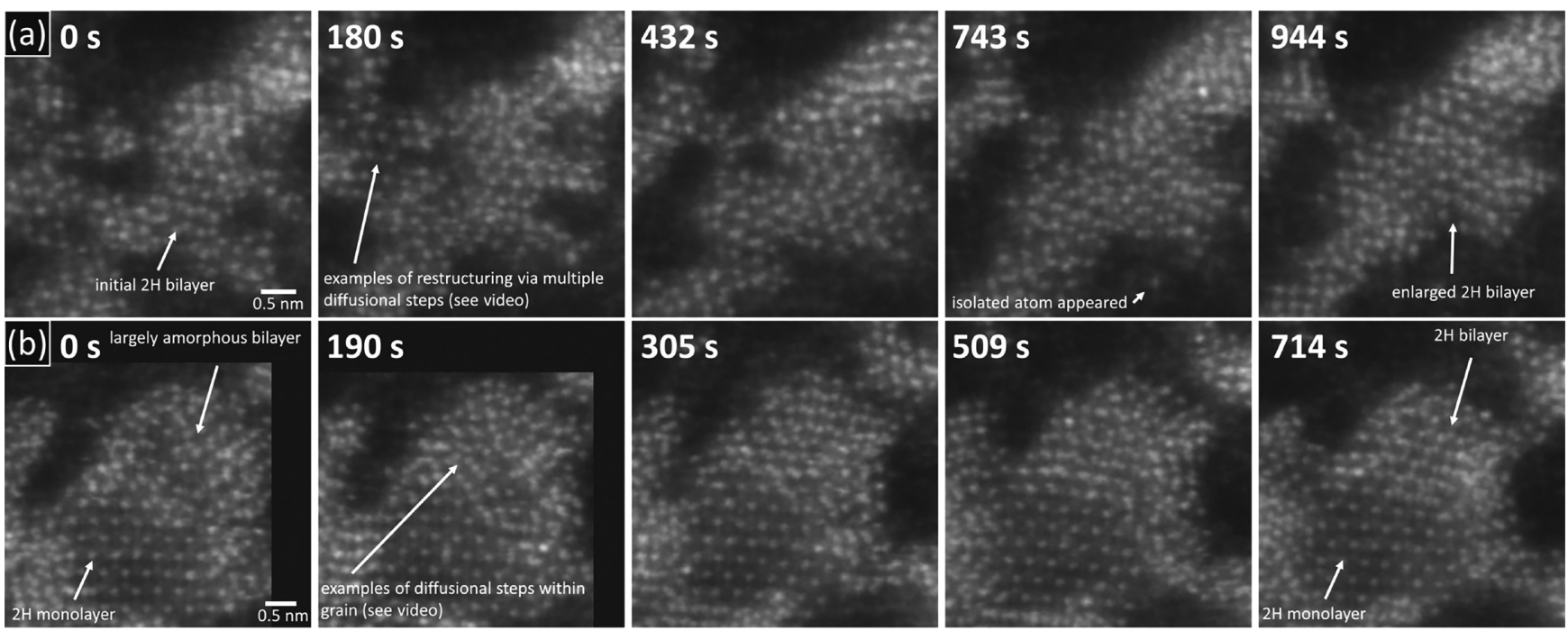

Figure 5. (a,b) HAADF STEM image series of other locations from a $\sim 2 \mathrm{~nm} \mathrm{MoS}_{2}$ on graphene during continuous e-beam exposure (relative time stamps indicated). The corresponding HAADF STEM in situ videos taken during the continuous e-beam exposure (temporal resolution $\sim 2.7 \mathrm{~s}$ per frame) for (a) and (b) are given in Video S1 and Video S2, respectively (time lapsed to 4 frames per second, time stamps indicated for salient frames in the videos). 
respectively. Based on this identification of a $2 \mathrm{H}$ monolayer $\mathrm{MoS}_{2}$ region, we recalculate the HAADF intensity counts in Figure $3 \mathrm{a}$ to a relative intensity with respect to the HAADF intensity from a single $S$ atom as shown in the false color coded image in Figure $3 c$, in which a single $S$ atom $\left(Z_{S}=16\right)$ has relative intensity 1 and a single Mo atom $\left(Z_{\mathrm{Mo}}=42\right)$ has a relative intensity of $\sim 4.9$. In doing so we establish a straightforward way of identifying the structure of further $\mathrm{MoS}_{2}$ regions in our in situ STEM data. For increasing layer numbers, the spatial average intensity over a region scales approximately linearly with number of layers. The atomic stacking type in such multilayers can then in turn be discerned by further analyzing the spatially resolved intensities as a function of atomic positions. For instance, the region left of the monolayer patch in Figure $3 \mathrm{c}$ is thereby consistent with a $2 \mathrm{H}$ bilayer, as indicated in the image. Furthermore, in Figure $3 \mathrm{c}$ several isolated Mo atoms can be identified on the graphene support (see labeled examples) as well as one Mo adatom on the $2 \mathrm{H}$ bilayer patch (correspondingly labeled).

Following this method, we present in Figure 4 the recalculated data from the time series in Figure 1 and identify via the spots (a) to (g) labeled in Figure 4 three salient structural evolution processes that we find to occur in parallel in this image series. Additional HAADF STEM time series data in Figure 5 for two other regions on a $\sim 2 \mathrm{~nm} \mathrm{MoS}_{2}$ on graphene sample show a matching evolution. Importantly, for Figure 5a,b we also provide the corresponding HAADF STEM in situ videos taken during the continuous e-beam exposure (temporal resolution $\sim 2.7 \mathrm{~s}$ per frame) as Video S1 and Video S2, respectively.

In Figure 4 spots (a) and (b) we follow the structural evolution that we most commonly observe upon e-beaminduced crystallization: An initially amorphous region crystallizes into bilayer patches of $2 \mathrm{H} \mathrm{MoS}_{2} \cdot{ }^{16}$ In particular, for spot (a) we observe after $3 \mathrm{~min}$ nucleation of a $2 \mathrm{H}$ bilayer patch in the upper right. This region has expanded after $8 \mathrm{~min}$, whereupon at $14 \mathrm{~min}$, two more nonconnected $2 \mathrm{H}$ bilayer regions appeared in the lower left and lower right of spot (a). From 8 to $15 \mathrm{~min}$, these regions restructure, including some intermittent shrinking, and before $17 \mathrm{~min}$, the $2 \mathrm{H}$ bilayer patches have expanded into one connected single crystalline grain. This grain in spot (a) at 17 min covers $\sim 5.7 \mathrm{~nm}^{2}$, which is the largest connected single crystalline grain imaged in Figures 1 and 4. A similar evolution is also found in Figure 5 and Video S1 and Video S2: In Figure 5a/Video S1, a small 2H bilayer nucleus near the center of the image grows in lateral size at the expense of surrounding amorphous $\mathrm{MoS}_{2}$ deposits on the graphene. In Figure 5b/Video $\mathrm{S} 2$, a $2 \mathrm{H}$ monolayer is observed in the center of the frame with an adjacent largely amorphous bilayer region to the upper right. Upon continued e-beam exposure, this amorphous bilayer region crystallizes into a larger $2 \mathrm{H}$ bilayer grain.

This generally observed preferential formation of the $2 \mathrm{H}$ phase from a- $\mathrm{MoS}_{2}$ confirms previous formation energy calculations of various $\mathrm{MoS}_{2}$ bulk polymorphs that predicted $2 \mathrm{H}$ to be the energetically most favored structure. ${ }^{11,18}$ Since in our ultrathin $\mathrm{MoS}_{2}$-graphene heterostructures thermodynamic bulk properties may be modified by effects from heterogeneous interfaces and free surfaces etc., ${ }^{56}$ we model in Figure S4 nonbulk representations of heterostructures of a-MoS $\mathrm{M}_{2}$ on graphene in comparison to a crystalline $2 \mathrm{H} \mathrm{MoS}_{2}$ bilayer patch on graphene and calculate their formation energies using density functional theory (DFT). From our calculations, we find the crystalline $2 \mathrm{H}$ bilayer $\mathrm{MoS}_{2}$ patch on graphene to be between $\sim 0.26 \mathrm{eV} /$ atom and $\sim 0.34 \mathrm{eV}$ /atom lower in formation energy than the corresponding a- $\mathrm{MoS}_{2}$ on graphene. This suggests (in general agreement with previous bulk calculations $)^{11,18}$ also for our ultrathin $\mathrm{MoS}_{2}$-graphene heterostructures that a thermodynamic driving force is behind the experimentally observed crystallization of a- $\mathrm{MoS}_{2}$ to $2 \mathrm{H}$ $\mathrm{MoS}_{2}$, whereby we hypothesize that the energy input ${ }^{6}$ from the scanning e-beam is helping to overcome kinetic barriers ${ }^{57}$ to crystallization.

In contrast to this theoretically predicted evolution of our a$\mathrm{MoS}_{2}$ toward $2 \mathrm{H}$, we however find in Figure 4 in spot (c) in the lower right at $17 \mathrm{~min}$ a crystalline $\mathrm{MoS}_{2}$ region to have evolved from initial a- $\mathrm{MoS}_{2}$ that has a spatial average intensity consistent with bilayer, but where the intensities as a function of atomic positions indicate that this bilayer patch is not of the $2 \mathrm{H}$ type. Instead the measured atomically resolved intensity profile of spot (c) at $17 \mathrm{~min}$ is consistent with a bilayer that shows homonuclear stacking (either $2 \mathrm{H}^{\prime}$ or $1 \mathrm{H}$ ), ${ }^{17}$ where Mo atoms of the second layer are placed directly above Mo atoms of the first layer. This observation of homonuclear stacking next to $2 \mathrm{H}$ stacking suggests that, besides crystallization toward equilibrium $2 \mathrm{H}$, initial a- $\mathrm{MoS}_{2}$ can also crystallize into other $\mathrm{MoS}_{2}$ polymorphs under fixed processing conditions, thereby resulting in co-existence of several $\mathrm{MoS}_{2}$ polymorphs. While homonuclear stacking is energetically not favored, ${ }^{18}$ it has been previously observed ex situ in annealed liquid-phase exfoliated $\mathrm{MoS}_{2}$ layers, ${ }^{17}$ where similar to our observation here, equilibrium $2 \mathrm{H}$ bilayers and nonequilibrium homonuclearly stacked bilayer regions co-existed. We note that this resemblance between our in situ and previous ex situ data implies that our atomic-scale in situ observations are indeed capturing processes which are relevant to ex situ $\mathrm{MoS}_{2}$ processing.

Besides predominant $2 \mathrm{H}$ stacking and homonuclear stacking, we find after extended e-beam exposure (17 $\mathrm{min})$ also a third salient stacking type shown in Figure 4 at the spots labeled (d). Compared to the $2 \mathrm{H}$ and homonuclear bilayers, this region exhibits no six-fold symmetry but a line appearance of different symmetry. Measuring characteristic distances for spots (d) in Figure 4, we find this structure to exhibit a spacing of $\sim 0.23 \mathrm{~nm}$ which is comparably shrunk from the typical $\sim 0.26 \mathrm{~nm}$ distance in $2 \mathrm{H} \mathrm{MoS}$. This structure is thereby reminiscent of merging line defects in $\mathrm{MoS}_{2}$ layers that result from loss of $\mathrm{S}$ under continued e-beam illumination. ${ }^{50}$ Such a $\sim 0.23 \mathrm{~nm}$ fringe spacing is also approaching the spacings expected for metallic Mo phases, ${ }^{58}$ and the observed line-like symmetry is also evocative of previously reported S-deficient $\mathrm{MoS}_{2-x}$ phases. ${ }^{59}$ For these reasons we tentatively assign the structure at spots (d) in Figure 4 to locally S-deficient $\mathrm{MoS}_{2-x}$, which is created during our continued e-beam exposure in the STEM by $S$ loss from the initially present $\mathrm{MoS}_{2}$. This $\mathrm{S}$ loss leads to crystallization/restructuring not toward a $\mathrm{MoS}_{2}$ polymorph but a S-deficient structure, akin to recent results on e-beam-induced S-deficient phase formation in $\mathrm{SnS}_{2}{ }^{60}$ We note that controlled ex situ formation of such S-deficient $\mathrm{MoS}_{2-x}$ has previously been suggested to be beneficial for certain applications requiring $2 \mathrm{D}$ Mo-S compounds with increased reactivity. ${ }^{61} \mathrm{We}$ also note however that our EDX measurements in the TEM in Figure S2, discussed above, as well as the observation in Figure 4 that globally the $2 \mathrm{H} \mathrm{MoS}$ phase is the predominant phase suggests that on a larger scale, 
the loss of $\mathrm{S}$ is limited at $60 \mathrm{kV}$ for our in situ crystallization conditions.

Having established the three salient Mo-S structures in our data, we note that the observed $\mathrm{MoS}_{2}$ crystallization and restructuring processes under the e-beam are found to be highly dynamic: Notably, in spot (e) in Figure 4 (corresponding also to the region shown in Figure 3), the small bilayerthick region toward the left in Figure 4/spot (e) evolves from an amorphous island with approximate bilayer thickness (0 $\mathrm{min})$ to a crystalline bilayer with $2 \mathrm{H}$ stacking $(14 \mathrm{~min})$. This $2 \mathrm{H}$ bilayer then intermittently evolves to homonuclear bilayer stacking $(15 \mathrm{~min})$ only to then return to $2 \mathrm{H}$-type stacking ( 17 min and $18 \mathrm{~min}$ in Figure 3 ). This time-dependent appearance/disappearance of $\mathrm{MoS}_{2}$ polymorphs indicates that various polymorphs can not only co-evolve but also dynamically transform into each other during processing. Similarly, the in situ e-beam exposure videos (Video S1 and Video S2, corresponding to Figure 5) indicate a highly dynamic local evolution during the overall a-MoS 2 to $\mathrm{nc}-\mathrm{MoS}_{2}$ crystallization, where in particular the emerging $2 \mathrm{H}$ bilayer grains are far from static but exhibit alternating growth and shrinkage periods. A key question behind such dynamics is the underlying mechanism of atomic movement. In this context, currently little is known about the diffusing moieties in $\mathrm{MoS}_{2}$ during crystallization and restructuring. ${ }^{62-64}$ This results from the difficulty of their direct observation due to their presumably fast diffusion speeds. ${ }^{37}$ While even the best time resolution in our data during the continuous e-beam exposure in situ videos ( $2.7 \mathrm{~s}$ per frame, as shown in Video S1 and Video S2, corresponding to Figure 5) is insufficient to directly observe diffusing species, close inspection of our STEM data can give hints of the diffusing species in our e-beam-induced $\mathrm{MoS}_{2}$ restructuring. We note that some of the adventitious carbon residues on the bare graphene areas in Figure 1a, Figure 5, Video S1, and Video S2 can act as intermediate traps for species diffusing over the graphene, thus allowing to draw some preliminary conclusions about moieties diffusing between $\mathrm{MoS}_{2}$ clusters on the graphene: Our element-specific HAADF data identify isolated Mo atoms on the graphene support (some examples labeled in Figure $3 \mathrm{c}$ and as spots (f) in Figure 4) that change their location and attach/detach from larger $\mathrm{MoS}_{2}$ structures during the time series in Figures 3 and 4. Such suspected diffusion of Mo atoms between $\mathrm{MoS}_{2}$ clusters on graphene is also consistent with Figure 5a/Video $S 1$ where we also observe at better temporal resolution in the in situ video multiple instances of positional changes of Mo atoms during e-beam exposure that lead to overall mass transport from one $\mathrm{MoS}_{2}$ cluster to another across the graphene. An example of this is the evolution of a "neck" between two eventual $\mathrm{MoS}_{2}$ clusters visible left of the image center in Video S1 (location of forming neck indicated in Figure $5 \mathrm{a} / 180 \mathrm{~s}$ by a white arrow). Another example is the appearance and diffusional movement of several isolated Mo atoms in Video $\mathrm{S} 1$ below the $2 \mathrm{H}$ bilayer cluster (location indicated in Figure 5a/743 s by a white arrow). As such our data indicate that some Mo mass transport is occurring between $\mathrm{MoS}_{2}$ clusters across the graphene support during a$\mathrm{MoS}_{2}$ crystallization and restructuring. Given the lower atomic number of $S$ atoms, clear identification of isolated $S$ on the basal plane of the graphene support next to adventitious carbon adsorbates is more challenging in our data. We have however labeled as spots (g) in Figure 4 some candidates that may be attributed to single $S$ atoms on the graphene basal plane, which would suggest that also isolated $\mathrm{S}$ atoms are diffusing over the graphene during the restructuring. Besides longer range mass transport between adjacent grains, a second type of diffusion during the restructuring is short-range diffusion of atoms within a given grain. An example of such diffusion events within a grain is found in Figure $3 c$ where a Mo adatom is intermittently located on a $\mathrm{MoS}_{2}$ bilayer patch, consistent with a recently identified ${ }^{64}$ metastable adatom configuration on a $\mathrm{MoS}_{2}$ lattice. The in situ data in Video S2 further shows multiple instances of diffusional steps and positional changes between adjacent atoms within a given bilayer $\mathrm{MoS}_{2}$ grain during its crystallization from a-MoS 2 to $2 \mathrm{H}$ $\mathrm{MoS}_{2}$ (location indicated by white arrow in Figure 5b/190 s). Thereby our data show that such short-range diffusion events within a given grain are another major mechanism of crystallization and restructuring of a- $\mathrm{MoS}_{2}$ to $\mathrm{nc}-\mathrm{MoS}_{2}$.

After close inspection of atomically resolved information, we quantitatively analyze the data in Figures 1 and 4 with respect to the visual notion of a-MoS $\mathrm{M}_{2}$ dewetting from the graphene support during its crystallization to nc- $\mathrm{MoS}_{2}$ on wider scale: The analysis in Figure S5 shows that for the STEM data in Figure 1a from 0 to $15 \mathrm{~min}$, the bare graphene area notably increases, while, conversely, $\mathrm{MoS}_{2}$ regions with monolayer and submonolayer $\mathrm{MoS}_{2}$ coverage reduce and $\mathrm{MoS}_{2}$ regions with bi- and trilayer coverage slightly increase in area. This confirms the visual impression that the low coverage a- $\mathrm{MoS}_{2}$ clusters dewet from the graphene support and the thus released Mo and $S$ attaches on average to thicker $\mathrm{MoS}_{2}$ regions. Previous theoretical work has predicted (based on considerations of edge energies and interlayer binding in $\mathrm{nc}-\mathrm{MoS}_{2}$ clusters) an increasing equilibrium average layer number for $\mathrm{MoS}_{2}$ crystallites with increasing lateral size. ${ }^{29}$ For our data, this would suggest that our $\mathrm{MoS}_{2}$ clusters possibly transform toward their equilibrium thickness/lateral size ratio by the observed dewetting process via the energy input from the ebeam.

Our atomic-scale in situ observations during crystallization and restructuring of $\mathrm{MoS}_{2}$ have so far elucidated two key points: First, various $\mathrm{MoS}_{2}$ polymorphs can co-exist and evolve in parallel for fixed processing conditions. This links directly with previous ex situ reports on in-layer polymorphism ${ }^{12-15}$ and co-existence of various stacking types ${ }^{17,18,21,22}$ in $\mathrm{ex}$ situ processed $\mathrm{MoS}_{2}$, including chemical synthesis and CVD. As a second and equally important point, our in situ data now clarify that the structural evolution of the $\mathrm{MoS}_{2}$ leading to such polymorphism is not static but highly dynamic, where phases appear/disappear and transform into each other over time. Observation of such dynamics intrinsically requires an in situ approach as employed here.

While in our experiments the monolayer graphene onto which the a-MoS $\mathrm{M}_{2}$ is deposited onto is primarily employed as substrate for high-resolution STEM, ${ }^{5}$ the many emerging applications of vertical $\mathrm{MoS}_{2}$ /graphene heterostructures in energy, (opto-)electronics, and catalysis ${ }^{10,65-67}$ make also the properties of this $\mathrm{MoS}_{2} /$ graphene heterostructure interesting as such. A key drawback toward their elucidation via the data presented in Figures $1-5$ is however that the lattice of the supporting graphene is not resolved in these images due to nonoptimal imaging conditions for the lighter carbon $\left(Z_{\mathrm{C}}=6\right)$ as well as static residual adventitious carbon contamination which is typical ${ }^{5,36,68}$ for graphene samples from sample transport and storage in air. This precludes the assessment of orientational relations between the underlying graphene and 
the crystallizing $\mathrm{MoS}_{2}$ in Figures 1-4, despite the interesting observation that after $17 \mathrm{~min}$ electron beam exposure, the FT in Figure $1 \mathrm{~b}$ shows signs of one discrete hexagonal spot pattern across several nonconnected $\mathrm{MoS}_{2}$ crystallites. Such a discrete six-fold FT pattern would suggest a dominant orientation of the crystallized $\mathrm{MoS}_{2}$ that in turn opens the interesting question whether this dominant orientation may be related to a possible epitaxial relationship of the $\mathrm{MoS}_{2}$ to the underlying graphene support. Previous literature suggested that the nonexistence $\mathrm{c}^{22,69,70}$ or existence $\mathrm{c}^{22,71,72}$ of $\mathrm{MoS}_{2} /$ graphene epitaxy is highly process parameter dependent, resulting from the rather weak van der Waals interaction between $\mathrm{MoS}_{2}$ and graphene. ${ }^{69}$ When $\mathrm{MoS}_{2} /$ graphene epitaxy was found in previous work, rotational misalignment distributions peaked at $0^{\circ}$ and $30^{\circ} \cdot 22,71,72$

To resolve a possible orientation relation between the graphene support and the crystallizing nc- $\mathrm{MoS}_{2}$ domains under our conditions, we present the e-beam crystallization sequence in Figure 6a. In this series the graphene support in as-deposited

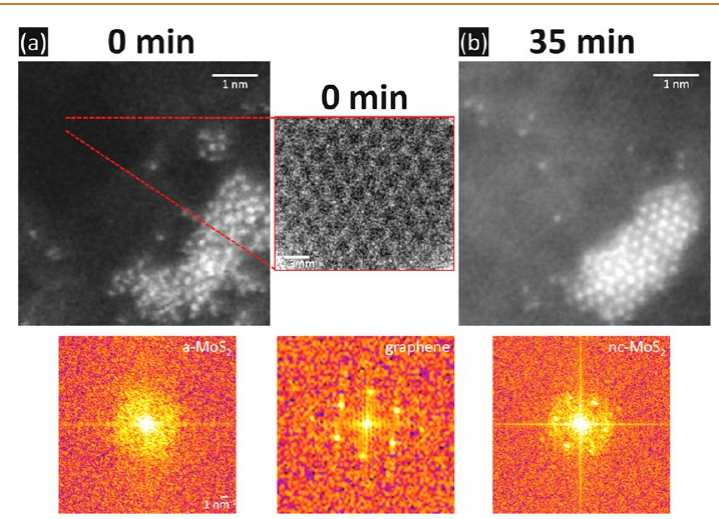

Figure 6. (a) HAADF STEM image of $\sim 2 \mathrm{~nm}$-thick $\mathrm{MoS}_{2}$ on graphene before continuous e-beam exposure $(0 \mathrm{~min})$. The corresponding FT underneath (a) is consistent with a-MoS $\mathrm{S}_{2}$. The inset in the middle shows a (medium angle annular dark field) close-up of an atomically clean graphene area to resolve the supporting graphene lattice and its orientation by the FT under the inset. (b) HAADF STEM image of the same location as (a) after 35 min continuous e-beam exposure. The FT underneath (b) reveals that the a-MoS $\mathrm{S}_{2}$ has crystallized under the e-beam to a single nc- $\mathrm{MoS}_{2}$ grain, which is misoriented to the graphene lattice directions seen in (a) by a rotation of $\sim 30^{\circ}$.

state ( 0 min e-beam exposure) shows both adventitious carbon covered but also atomically clean graphene areas. In the image center of the latter, the six-fold lattice of a single crystalline graphene region can be well resolved (inset) and its orientation can be straightforwardly discerned from the corresponding FT pattern below the inset. The $\mathrm{MoS}_{2}$ in Figure 6a is fully amorphous in its as-deposited state, consistent with our findings above. During continuous e-beam exposure, two processes happen, resulting in Figure $6 \mathrm{~b}$ which shows the same region after $35 \mathrm{~min}$ of e-beam exposure: (i) Same as in Figure 1 , the e-beam exposure leads to crystallization of the a-MoS 2 to nc-MoS 2 with $\mathrm{MoS}_{2}$ layers parallel to the graphene support; and (ii) concurrently, adventitious carbon diffusion into the field of view (typical for extended STEM imaging) ${ }^{5}$ obscures the initially atomically clean graphene area in the center. Nevertheless, the FT of the nc-MoS 2 in Figure $6 \mathrm{~b}$ now allows to assess the orientation of the crystallized $\mathrm{MoS}_{2}$ layers. Assuming that the graphene lattice in Figure 6a extends across the entire field of view (which is a reasonable assumption given the typically $\mu \mathrm{m}$-sized graphene domains in such polycrystalline CVD graphene), ${ }^{73,74}$ we can therefore by comparison of the FTs in Figure 6a,b (graphene and $\mathrm{nc}-\mathrm{MoS}_{2}$, respectively) measure the misorientation of the crystallized $\mathrm{MoS}_{2}$ domains and the underlying graphene lattice. We find for the data in Figure 6 a misorientation of $\sim 30^{\circ}$ which is consistent with previously reported epitaxial misorientation values for vertical $\mathrm{MoS}_{2} /$ graphene heterostructures. ${ }^{71,72}$ Combined with the development of one discrete hexagonal spot pattern over several nc- $\mathrm{MoS}_{2}$ islands across the entire field of view in Figure 1 , this is indicative that an epitaxial interaction between the graphene support and the crystallizing $\mathrm{MoS}_{2}$ can also prevail under our STEM conditions. We note, however, that when considering e-beam-induced crystallization in TEM at a larger field of view (up to $900 \mathrm{~nm}$ ) in Figures S1-S3, we find that on the single crystalline graphene grains rings which are typical of in-plane randomly rotated polycrystalline $\mathrm{nc}-\mathrm{MoS}_{2}$ are produced instead of discrete $\mathrm{MoS}_{2}$ patterns. Such polycrystallinity over a large field of view is inconsistent with a strong epitaxial interaction. The combination of our STEM and TEM results therefore suggests that the driving force toward $\mathrm{MoS}_{2} /$ graphene epitaxy under our conditions is comparably weak and epitaxy can prevail under certain conditions (as in STEM) but is easily overridden (as in TEM) by other factors, leading to epitaxial or nonepitaxial growth depending on exact processing conditions and kinetics. This is in line with the processdependent results on $\mathrm{MoS}_{2} /$ graphene heterostructure epitaxy in previous ex situ reports. $22,69-72$

Our data of the in-plane e-beam-induced crystallization have so far been limited to studying atomically thin a-MoS films ( $\sim 2 \mathrm{~nm}$ nominal thickness). However, both for electronic and catalytic applications thicker $\mathrm{MoS}_{2}$ films are also under investigation. $^{30,34,75}$ Figure 7 therefore presents time-resolved STEM measurements on comparatively thicker a-MoS films of $\sim 10 \mathrm{~nm}$ nominal thickness. In particular, we are comparing in Figure 7 a region which was partly shadowed during $\mathrm{MoS}_{2}$ deposition and is therefore of somewhat lower thickness (darker HAADF signal in the central region of Figure 7a) with regions consisting of the full deposited $\sim 10 \mathrm{~nm}$ nominal thickness (bright HAADF signal at the left and right edges of Figure 7a). In keeping with our data for the thinner $\mathrm{MoS}_{2}$ films above, the thin region in the center of Figure $7 \mathrm{a}$ shows no inplane order for the as-deposited films $(0 \mathrm{~min}$ e-beam illumination) and is consistent with a-MoS . Similarly, the thicker regions toward the left and right edges of Figure $7 \mathrm{a}$ are largely amorphous in their visual appearance in the asdeposited state $(0 \mathrm{~min})$. This is also corroborated by the corresponding FT in Figure $7 \mathrm{~b}(0 \mathrm{~min})$. We note, however, that on the left side in the thicker region in Figure $7 \mathrm{a}(0 \mathrm{~min})$, two pronounced lattice fringes with a spacing of $\sim 0.6 \mathrm{~nm}$ are visible. Such $\sim 0.6 \mathrm{~nm}$ layer spacing is indicative of the (002) layer distance in $\mathrm{MoS}_{2}$, therefore suggesting an image interpretation of $\mathrm{MoS}_{2}$ planes being parallel to the e-beam and thereby being perpendicular to the graphene support.

Upon continued e-beam exposure we find clear signs of crystallization for the thicker a-MoS . As above, the thinner regions of Figure 7a crystallize with $\mathrm{MoS}_{2}$ layers parallel to the graphene support $(22$ and $34 \mathrm{~min}$, as also shown at higher magnification in Figure $7 c, d)$. Concurrently and unlike the thinner films above, in the thicker $\mathrm{MoS}_{2}$ regions, multiple sets of $\sim 0.6 \mathrm{~nm}$ lattice fringes appear upon e-beam exposure. These sets of $\sim 0.6 \mathrm{~nm}$ fringes each consist of $\sim 3$ to $\sim 9$ fringes 


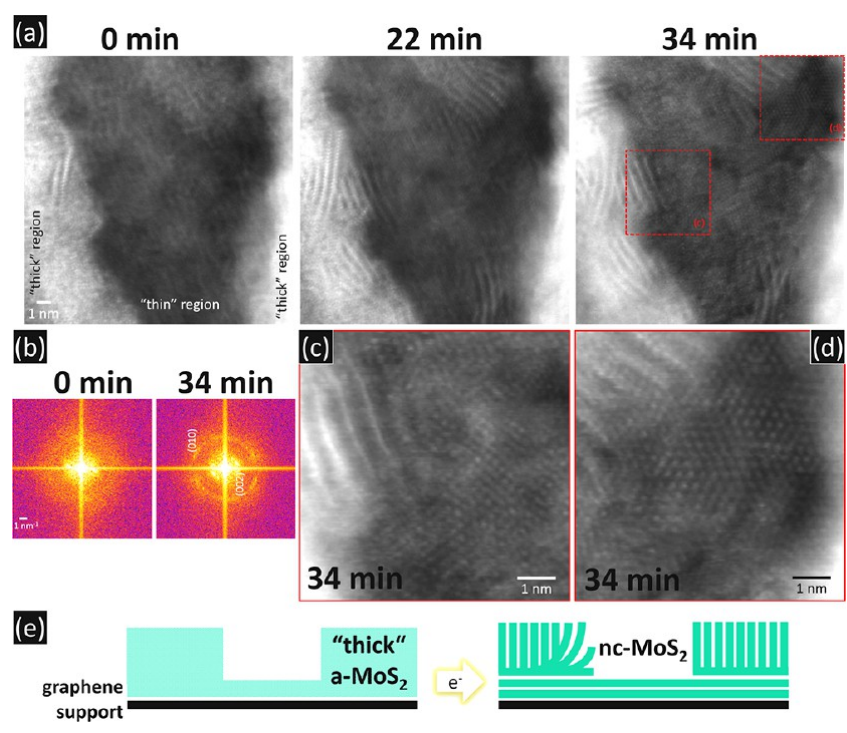

Figure 7. (a) HAADF STEM image series of $\sim 10 \mathrm{~nm} \mathrm{MoS}$ on graphene during continuous e-beam exposure (time stamps indicated). In particular we show in the image center a region that was partly shadowed during $\mathrm{MoS}_{2}$ deposition and is therefore of lower thickness (darker HAADF signal) and compare it with regions corresponding to the full $\sim 10 \mathrm{~nm}$ nominal thickness on the image's left and right edges (bright HAADF signal). (b) FTs of (a) with corresponding time stamps indicated. (c,d) Close-ups of (a), as indicated by red frames. (e) Schematic illustration summarizing the observations deduced from (a).

parallel to each other, while the individual sets are rotated in plane with respect to each other. The appearance of such sets of $\sim 0.6 \mathrm{~nm}$ fringes is a clear sign of crystallization of the initial a- $-\mathrm{MoS}_{2}$ in the thicker regions to nc- $\mathrm{MoS}_{2}$ with the $\mathrm{MoS}_{2}$ layers in the direction perpendicular to the graphene support. This is also well reflected in the FT data in Figure $7 \mathrm{~b}(34 \mathrm{~min})$ that shows the corresponding $\mathrm{MoS}_{2}$ (002) reflections (which are naturally missing in the FTs of the thin $\mathrm{MoS}_{2}$ which crystallized with the layers parallel to the support in Figure 1b). The emergence of $\mathrm{MoS}_{2}$ (002) reflections in the $\sim 10 \mathrm{~nm}$ $\mathrm{MoS}_{2}$ films upon e-beam exposure is also corroborated by TEM measurements at a larger field of view, shown in Figure S6. Importantly, further inspection of the STEM data in Figure $7 \mathrm{a}(34 \mathrm{~min})$ and Figure $7 \mathrm{~d}$ shows that under the $\sim 0.6 \mathrm{~nm}$ fringe sets, an in-plane ordered $\mathrm{MoS}_{2}$ lattice continues. This leads to the interpretation of the data in Figure 7 that thicker $\mathrm{MoS}_{2}$ regions ( $\sim 10 \mathrm{~nm}$ nominal thickness) crystallize during ebeam exposure in a two-segment morphology: The first few $\mathrm{MoS}_{2}$ layers near the support interface crystallize parallel to the graphene support (same as the thinner $\sim 2 \mathrm{~nm} \mathrm{MoS}$ regions in Figure 1) but then farther away from the graphene support the direction of the evolving $\mathrm{MoS}_{2}$ layer orientation changes for the thicker films, resulting in further $\mathrm{MoS}_{2}$ to crystallize with its layers perpendicular to their support (i.e., perpendicular to the graphene support and the first few $\mathrm{MoS}_{2}$ layers). We note that these layers with overall perpendicular orientation may also partly be curved along their length. ${ }^{37}$ Figure $7 \mathrm{e}$ schematically illustrates the evolution of this suggested two-segment nc$\mathrm{MoS}_{2}$ film structure with in-plane crystallization near the support interface and out-of-plane crystallization beyond for thicker a- $\mathrm{MoS}_{2}$ films. Previous literature has found both parallel and perpendicular layer growth in thicker $\mathrm{MoS}_{2}$ films depending on exact synthesis conditions. ${ }^{37,76}$ Importantly we note that the observation of our two-segment morphology is in excellent agreement with previous ex situ studies on annealed PVD $\mathrm{MoS}_{2}$ films, ${ }^{30,77}$ that is, films that were deposited and processed under similar deposition condition as ours, in which the same two-segment morphology was reported.

\section{CONCLUSIONS}

In summary, our work provides in situ atomic-scale observations of the crystallization and restructuring of the important TMDC $\mathrm{MoS}_{2}$. Our data elucidate the complex evolution of a material with such pronounced and, as we show, dynamic polymorphism. Our observations thereby visualize multiple fundamental processes that are underlying the varied $\mathrm{MoS}_{2}$ morphologies obtained in previous ex situ $\mathrm{MoS}_{2}$ processing studies. Our beam-driven in situ imaging and materials modification approach can be expected to be extendable to several other TMDCs and 2D materials that crystallize equally easily as $\mathrm{MoS}_{2}$ (i.e., $2 \mathrm{D}$ materials that can grow without the requirements for high processing temperatures $^{2}$ and for a thick process catalyst $\left.{ }^{1}\right)$. We expect that our here presented in situ methodology will contribute toward an improved fundamental atomic-scale understanding of TMDC and $2 \mathrm{D}$ materials synthesis and integration processing.

\section{METHODS}

Samples for high-resolution STEM were prepared as follows: First continuous monolayer graphene films were grown by $\mathrm{CVD}$ on $\mathrm{Cu}$ catalysts ${ }^{74}$ in a $\mathrm{CH}_{4} / \mathrm{H}_{2} / \mathrm{Ar}$ mixture at $960{ }^{\circ} \mathrm{C}^{73}$ The graphene films were then suspended as membranes by transfer onto holey carbon-foil TEM grids with regular hole arrays (Quantifoil) using a polymer-free transfer process, ${ }^{41}$ which avoids the detrimental residues ${ }^{36,42,43}$ typically associated with polymer-based transfers and thus ensures an as clean as possible $\mathrm{MoS}_{2}$ /graphene interface from scalable processing. Onto these graphene covered TEM grids, PVD $\mathrm{MoS}_{2}$ (nominal thicknesses from $\sim 2 \mathrm{~nm}$ to $\sim 10 \mathrm{~nm}$ ) was then sputter deposited from a compound $\mathrm{MoS}_{2}$ target. During PVD, the samples were left at nominal room temperature (i.e., without intentional substrate heating applied). These conditions are known to lead to deposition of a-MoS ${ }^{34}$ Throughout and after fabrication, samples were stored and transported in ambient air.

STEM was measured in an aberration corrected Nion UltraSTEM 100 at an electron acceleration voltage of $60 \mathrm{kV}$, acquiring HAADF (80 to $200 \mathrm{mrad}$ ) data. The STEM data in Figures 1a, 2, 3a, 4, 5, 6, and $7 \mathrm{c}, \mathrm{d}$ have been Gaussian blurred ( 2 pixel radius) to improve visibility. Typical beam currents during STEM imaging of $\sim 30 \mathrm{pA}$ result for spot sizes of $\sim 1 \AA^{2}$ in electron dose rates directly under the beam of $\sim 5 \times 10^{8} \mathrm{e}^{-} \AA^{-2} \mathrm{~s}^{-1}$, which in turn equate to average dose rates of $\sim 5 \times 10^{4} \mathrm{e}^{-} \AA^{-2} \mathrm{~s}^{-1}$ for continuous scanning of a $10 \mathrm{~nm} \times 10$ $\mathrm{nm}$ area as in Figure 1. For the crystallization series in STEM, continuous e-beam exposure was achieved via continuous STEM scanning. We note that for imaging at a wider field of view/lower dose rates in the STEM, the e-beam-induced crystallization correspondingly proceeds less pronounced. In order to minimize reactions with residual gas species during STEM imaging, the employed STEM column leaves the sample in a vacuum of $\sim 10^{-9}$ mbar during imaging. During STEM imaging samples were not intentionally heated. Note that all samples were annealed at $\sim 140^{\circ} \mathrm{C}$ in a vacuum of $10^{-5} \mathrm{mbar}$ for $\sim 8 \mathrm{~h}$ prior to loading into the STEM in order to desorb adventitious hydrocarbons and adsorbed water from sample storage in ambient. We crosscheck by TEM and SAED without preheating that this low-temperature vacuum bake did not result in any significant a$\mathrm{MoS}_{2}$ crystallization. BF-TEM and SAED at $80 \mathrm{kV}$ and $200 \mathrm{kV}$ electron acceleration voltage were measured in a Philips CM200 TEM with the sample in a vacuum of $\sim 10^{-6}$ mbar. In the CM200 TEM, a wide e-beam was used for imaging and SAED at electron dose rates $\left(\sim 4 \times 10^{1} \mathrm{e}^{-} \AA^{-2} \mathrm{~s}^{-1}\right)$ that did not induce a-MoS 2 crystallization. In order to induce a-MoS $\mathrm{S}_{2}$ crystallization in the CM200 TEM, the e- 
beam was focused to achieve electron dose rates of $\sim 3 \times 10^{3} \mathrm{e}^{-} \AA^{-2}$ $\mathrm{s}^{-1}$. SAED at $60 \mathrm{kV}$ electron acceleration voltage was measured in a FEI Tecnai F20 TEM with a vacuum of $\sim 10^{-6} \mathrm{mbar}$ and beam current densities of $\sim 2 \times 10^{1} \mathrm{e}^{-} \AA^{-2} \mathrm{~s}^{-1}$ for imaging/SAED and of $\sim 1$ $\times 10^{3} \mathrm{e}^{-} \AA^{-2} \mathrm{~s}^{-1}$ to induce in situ crystallization. EDX was measured at $60 \mathrm{kV}$ in the F20 TEM with an EDAX Apollo XLTW SDD system. Elemental quantification from thus obtained EDX data of the a-MoS films was crosschecked by additional EDX measurements using an Oxford Instruments X-max system installed in a Zeiss Supra 55VP scanning electron microscope (SEM) operated at $20 \mathrm{kV}$ that was calibrated against mechanically exfoliated $\mathrm{MoS}_{2}$ reference crystals. Additional SEM-based EDX measurements on blanket a-MoS films deposited at identical conditions as the a- $\mathrm{MoS}_{2} /$ graphene heterostructures confirmed lateral homogeneity of stoichiometry of our a$\mathrm{MoS}_{2}$ films. For details on structural data analysis methodology and our DFT calculations see the Supporting Information.

\section{ASSOCIATED CONTENT}

\section{(5) Supporting Information}

The Supporting Information is available free of charge on the ACS Publications website at DOI: 10.1021/acsnano.8b04945.

Additional STEM data and analyses, EDX data, DFT calculations and details on methodology (PDF)

Video S1 (AVI)

Video S2 (AVI)

\section{AUTHOR INFORMATION}

\section{Corresponding Author}

*E-mail: bernhard.bayer@univie.ac.at.

\section{ORCID}

Bernhard C. Bayer: 0000-0002-4829-3207

Toma Susi: 0000-0003-2513-573X

Jani Kotakoski: 0000-0002-1301-5266

Dominik Eder: 0000-0002-5395-564X

Jannik C. Meyer: 0000-0003-4023-0778

\section{Notes}

The authors declare no competing financial interest.

\section{ACKNOWLEDGMENTS}

We acknowledge support from the Austrian Research Promotion Agency (FFG) under project 848152-GraphenMoFET. B.C.B. acknowledges funding from the European Union's Horizon 2020 research and innovation program under the Marie Skłodowska-Curie grant agreement 656214-2DInterFOX. M.R.A.M. and J.C.M. acknowledge support by the European Research Council starting grant no. 336453PICOMAT and T.S. under grant no. 756277-ATMEN. M.R.A.M. and J.K. acknowledge the Austrian Science Fund (FWF) for funding through project I3181-N36 and J.C.M. through project P25721-N20. We acknowledge use of the facilities at the University Service Centre for Transmission Electron Microscopy (USTEM), Vienna University of Technology, Austria for parts of this work.

\section{REFERENCES}

(1) Kling, J.; Hansen, T. W.; Wagner, J. B. Quantifying the Growth of Individual Graphene Layers by in Situ Environmental Transmission Electron Microscopy. Carbon 2016, 99, 261-266.

(2) Westenfelder, B.; Meyer, J. C.; Biskupek, J.; Kurasch, S.; Scholz, F.; Krill, C. E.; Kaiser, U. Transformations of Carbon Adsorbates on Graphene Substrates under Extreme Heat. Nano Lett. 2011, 11, 5123-5127.
(3) Liu, Z.; Lin, Y.-C.; Lu, C.-C.; Yeh, C.-H.; Chiu, P.-W.; Iijima, S.; Suenaga, K. In Situ Observation of Step-Edge in-Plane Growth of Graphene in a STEM. Nat. Commun. 2014, 5, 4055.

(4) Gong, C.; He, K.; Lee, G.-D.; Chen, Q.; Robertson, A. W.; Yoon, E.; Hong, S.; Warner, J. H. In Situ Atomic Level Dynamics of Heterogeneous Nucleation and Growth of Graphene from Inorganic Nanoparticle Seeds. ACS Nano 2016, 10, 9397-9410.

(5) Pantelic, R. S.; Meyer, J. C.; Kaiser, U.; Stahlberg, H. The Application of Graphene as a Sample Support in Transmission Electron Microscopy. Solid State Commun. 2012, 152, 1375-1382.

(6) Jesse, S.; He, Q.; Lupini, A. R.; Leonard, D. N.; Oxley, M. P.; Ovchinnikov, O.; Unocic, R. R.; Tselev, A.; Fuentes-Cabrera, M.; Sumpter, B. G.; Pennycook, S. J.; Kalinin, S. V.; Borisevich, A. Y. Atomic-Level Sculpting of Crystalline Oxides: Toward Bulk Nanofabrication with Single Atomic Plane Precision. Small 2015, 11, $5895-5900$

(7) Wang, Q. H.; Kalantar-Zadeh, K.; Kis, A.; Coleman, J. N.; Strano, M. S. Electronics and Optoelectronics of Two-Dimensional Transition Metal Dichalcogenides. Nat. Nanotechnol. 2012, 7, 699712 .

(8) Benck, J. D.; Hellstern, T. R.; Kibsgaard, J.; Chakthranont, P.; Jaramillo, T. F. Catalyzing the Hydrogen Evolution Reaction (HER) with Molybdenum Sulfide Nanomaterials. ACS Catal. 2014, 4, 39573971.

(9) Hansen, L. P.; Ramasse, Q. M.; Kisielowski, C.; Brorson, M.; Johnson, E.; Topsøe, H.; Helveg, S. Atomic-Scale Edge Structures on Industrial-Style MoS2 Nanocatalysts. Angew. Chem., Int. Ed. 2011, 50, 10153-10156.

(10) Koroteev, V. O.; Bulushev, D. A.; Chuvilin, A. L.; Okotrub, A. V.; Bulusheva, L. G. Nanometer-Sized MoS2 Clusters on Graphene Flakes for Catalytic Formic Acid Decomposition. ACS Catal. 2014, 4, 3950-3956.

(11) Lee, S. C.; Benck, J. D.; Tsai, C.; Park, J.; Koh, A. L.; AbildPedersen, F.; Jaramillo, T. F.; Sinclair, R. Chemical and Phase Evolution of Amorphous Molybdenum Sulfide Catalysts for Electrochemical Hydrogen Production. ACS Nano 2016, 10, 624-632.

(12) Eda, G.; Fujita, T.; Yamaguchi, H.; Voiry, D.; Chen, M.; Chhowalla, M. Coherent Atomic and Electronic Heterostructures of Single-Layer MoS2. ACS Nano 2012, 6, 7311-7317.

(13) Lin, Y.-C.; Dumcenco, D. O.; Huang, Y.-S.; Suenaga, K. Atomic Mechanism of the Semiconducting-to-Metallic Phase Transition in Single-Layered MoS2. Nat. Nanotechnol. 2014, 9, 391-396.

(14) Wang, X.; Shen, X.; Wang, Z.; Yu, R.; Chen, L. Atomic-Scale Clarification of Structural Transition of MoS2 upon Sodium Intercalation. ACS Nano 2014, 8, 11394-11400.

(15) Wang, Z.; Ning, S.; Fujita, T.; Hirata, A.; Chen, M. Unveiling Three-Dimensional Stacking Sequences of $1 \mathrm{~T}$ Phase MoS2Monolayers by Electron Diffraction. ACS Nano 2016, 10, 10308-10316.

(16) He, J.; Hummer, K.; Franchini, C. Stacking Effects on the Electronic and Optical Properties of Bilayer Transition Metal Dichalcogenides MoS 2, MoSe 2, WS 2, and WSe 2. Phys. Rev. B: Condens. Matter Mater. Phys. 2014, 89, 75409.

(17) Shmeliov, A.; Shannon, M.; Wang, P.; Kim, J. S.; Okunishi, E.; Nellist, P. D.; Dolui, K.; Sanvito, S.; Nicolosi, V. Unusual Stacking Variations in Liquid-Phase Exfoliated Transition Metal Dichalcogenides. ACS Nano 2014, 8, 3690-3699.

(18) Yan, A.; Chen, W.; Ophus, C.; Ciston, J.; Lin, Y.; Persson, K.; Zettl, A. Identifying Different Stacking Sequences in Few-Layer CVDGrown Mo S 2 by Low-Energy Atomic-Resolution Scanning Transmission Electron Microscopy. Phys. Rev. B: Condens. Matter Mater. Phys. 2016, 93, 41420.

(19) Lee, J.-U.; Kim, K.; Han, S.; Ryu, G. H.; Lee, Z.; Cheong, H. Raman Signatures of Polytypism in Molybdenum Disulfide. ACS Nano 2016, 10, 1948-1953.

(20) Xia, M.; Li, B.; Yin, K.; Capellini, G.; Niu, G.; Gong, Y.; Zhou, W.; Ajayan, P. M.; Xie, Y.-H. Spectroscopic Signatures of AA' and AB Stacking of Chemical Vapor Deposited Bilayer MoS2. ACS Nano 2015, 9, 12246-12254. 
(21) Enyashin, A. N.; Bar-Sadan, M.; Houben, L.; Seifert, G. Line Defects in Molybdenum Disulfide Layers. J. Phys. Chem. C 2013, 117, 10842-10848.

(22) Shi, Y.; Zhou, W.; Lu, A.-Y.; Fang, W.; Lee, Y.-H.; Hsu, A. L.; Kim, S. M.; Kim, K. K.; Yang, H. Y.; Li, L.-J.; et al. Van Der Waals Epitaxy of MoS2 Layers Using Graphene as Growth Templates. Nano Lett. 2012, 12, 2784-2791.

(23) Zhan, Y.; Liu, Z.; Najmaei, S.; Ajayan, P. M.; Lou, J. Large-Area Vapor-Phase Growth and Characterization of MoS2 Atomic Layers on a $\mathrm{SiO} 2$ Substrate. Small 2012, 8, 966-971.

(24) Najmaei, S.; Liu, Z.; Zhou, W.; Zou, X.; Shi, G.; Lei, S.; Yakobson, B. I.; Idrobo, J.-C.; Ajayan, P. M.; Lou, J. Vapour Phase Growth and Grain Boundary Structure of Molybdenum Disulphide Atomic Layers. Nat. Mater. 2013, 12, 754-759.

(25) van der Zande, A. M.; Huang, P. Y.; Chenet, D. A.; Berkelbach, T. C.; You, Y.; Lee, G.-H.; Heinz, T. F.; Reichman, D. R.; Muller, D. A.; Hone, J. C. Grains and Grain Boundaries in Highly Crystalline Monolayer Molybdenum Disulphide. Nat. Mater. 2013, 12, 554-561.

(26) Lee, Y.-H.; Zhang, X.-Q.; Zhang, W.; Chang, M.-T.; Lin, C.-T.; Chang, K.-D.; Yu, Y.-C.; Wang, J. T.-W.; Chang, C.-S.; Li, L.-J.; Lin, T.-W. Synthesis of Large-Area MoS2 Atomic Layers with Chemical Vapor Deposition. Adv. Mater. 2012, 24, 2320-2325.

(27) Kang, K.; Xie, S.; Huang, L.; Han, Y.; Huang, P. Y.; Mak, K. F.; Kim, C.-J.; Muller, D.; Park, J. High-Mobility Three-Atom-Thick Semiconducting Films with Wafer-Scale Homogeneity. Nature 2015, 520, 656-660.

(28) Elibol, K.; Susi, T.; O’Brien, M.; Bayer, B. C.; Pennycook, T. J.; McEvoy, N.; Duesberg, G. S.; Meyer, J. C.; Kotakoski, J. Grain Boundary-Mediated Nanopores in Molybdenum Disulfide Grown by Chemical Vapor Deposition. Nanoscale 2017, 9, 1591-1598.

(29) Cuddy, M. J.; Arkill, K. P.; Wang, Z. W.; Komsa, H.-P.; Krasheninnikov, A. V.; Palmer, R. E. Fabrication and Atomic Structure of Size-Selected, Layered MoS 2 Clusters for Catalysis. Nanoscale 2014, 6, 12463-12469.

(30) Ohashi, T.; Suda, K.; Ishihara, S.; Sawamoto, N.; Yamaguchi, S.; Matsuura, K.; Kakushima, K.; Sugii, N.; Nishiyama, A.; Kataoka, Y.; et al. Multi-Layered MoS2 Film Formed by High-Temperature Sputtering for Enhancement-Mode nMOSFETs. Jpn. J. Appl. Phys. 2015, 54, 04DN08.

(31) Tao, J.; Chai, J.; Lu, X.; Wong, L. M.; Wong, T. I.; Pan, J.; Xiong, Q.; Chi, D.; Wang, S. Growth of Wafer-Scale MoS 2 Monolayer by Magnetron Sputtering. Nanoscale 2015, 7, 2497-2503.

(32) Serrao, C. R.; Diamond, A. M.; Hsu, S.-L.; You, L.; Gadgil, S.; Clarkson, J.; Carraro, C.; Maboudian, R.; Hu, C.; Salahuddin, S. Highly Crystalline MoS2 Thin Films Grown by Pulsed Laser Deposition. Appl. Phys. Lett. 2015, 106, 52101.

(33) Serna, M. I.; Yoo, S. H.; Moreno, S.; Xi, Y.; Oviedo, J. P.; Choi, H.; Alshareef, H. N.; Kim, M. J.; Minary-Jolandan, M.; QuevedoLopez, M. A. Large-Area Deposition of MoS2 by Pulsed Laser Deposition with in Situ Thickness Control. ACS Nano 2016, 10, 6054-6061.

(34) Kaindl, R.; Bayer, B. C.; Resel, R.; Müller, T.; Skakalova, V.; Habler, G.; Abart, R.; Cherevan, A. S.; Eder, D.; Blatter, M.; Fischer, F.; Meyer, J. C.; Polyushkin, D. K.; Waldhauser, W. Growth, Structure and Stability of Sputter-Deposited MoS2 Thin Films. Beilstein J. Nanotechnol. 2017, 8, 1115-1126.

(35) Coleman, J. N.; Lotya, M.; O’Neill, A.; Bergin, S. D.; King, P. J.; Khan, U.; Young, K.; Gaucher, A.; De, S.; Smith, R. J.; et al. TwoDimensional Nanosheets Produced by Liquid Exfoliation of Layered Materials. Science 2011, 331, 568-571.

(36) Bayer, B. C.; Caneva, S.; Pennycook, T. J.; Kotakoski, J.; Mangler, C.; Hofmann, S.; Meyer, J. C. Introducing Overlapping Grain Boundaries in Chemical Vapor Deposited Hexagonal Boron Nitride Monolayer Films. ACS Nano 2017, 11, 4521-4527.

(37) Hansen, L. P.; Johnson, E.; Brorson, M.; Helveg, S. Growth Mechanism for Single-and Multi-Layer MoS2 Nanocrystals. J. Phys. Chem. C 2014, 118, 22768-22773.
(38) Fei, L.; Lei, S.; Zhang, W.-B.; Lu, W.; Lin, Z.; Lam, C. H.; Chai, Y.; Wang, Y. Direct TEM Observations of Growth Mechanisms of Two-Dimensional MoS2 Flakes. Nat. Commun. 2016, 7, 12206.

(39) Nguyen, D. N.; Nguyen, L. N.; Nguyen, P. D.; Thu, T. V.; Nguyen, A. D.; Tran, P. D. Crystallization of Amorphous Molybdenum Sulfide Induced by Electron or Laser Beam and Its Effect on H2-Evolving Activities. J. Phys. Chem. C 2016, 120, 2878928794.

(40) Dahl-Petersen, C.; Šarí́, M.; Brorson, M.; Moses, P. G.; Rossmeisl, J.; Lauritsen, J. V.; Helveg, S. Topotactic Growth of EdgeTerminated MoS2 from MoO2 Nanocrystals. ACS Nano 2018, 12, $5351-5358$

(41) Regan, W.; Alem, N.; Alemán, B.; Geng, B.; Girit, Ç.; Maserati, L.; Wang, F.; Crommie, M.; Zettl, A. A Direct Transfer of Layer-Area Graphene. Appl. Phys. Lett. 2010, 96, 113102.

(42) Kratzer, M.; Bayer, B. C.; Kidambi, P. R.; Matković, A.; Gajić, R.; Cabrero-Vilatela, A.; Weatherup, R. S.; Hofmann, S.; Teichert, C. Effects of Polymethylmethacrylate-Transfer Residues on the Growth of Organic Semiconductor Molecules on Chemical Vapor Deposited Graphene. Appl. Phys. Lett. 2015, 106, 103101.

(43) Elibol, K.; Bayer, B. C.; Hummel, S.; Kotakoski, J.; Argentero, G.; Meyer, J. C. Visualising the Strain Distribution in Suspended Two-Dimensional Materials under Local Deformation. Sci. Rep. 2016, 6, 28485.

(44) Komsa, H.-P.; Kotakoski, J.; Kurasch, S.; Lehtinen, O.; Kaiser, U.; Krasheninnikov, A. V. Two-Dimensional Transition Metal Dichalcogenides under Electron Irradiation: Defect Production and Doping. Phys. Rev. Lett. 2012, 109, 35503.

(45) Algara-Siller, G.; Kurasch, S.; Sedighi, M.; Lehtinen, O.; Kaiser, U. The Pristine Atomic Structure of MoS2Monolayer Protected from Electron Radiation Damage by Graphene. Appl. Phys. Lett. 2013, 103, 203107.

(46) Meyer, J. C.; Eder, F.; Kurasch, S.; Skakalova, V.; Kotakoski, J.; Park, H. J.; Roth, S.; Chuvilin, A.; Eyhusen, S.; Benner, G.; et al. Accurate Measurement of Electron Beam Induced Displacement Cross Sections for Single-Layer Graphene. Phys. Rev. Lett. 2012, 108, 196102.

(47) Komsa, H.-P.; Kurasch, S.; Lehtinen, O.; Kaiser, U.; Krasheninnikov, A. V. From Point to Extended Defects in TwoDimensional MoS 2: Evolution of Atomic Structure under Electron Irradiation. Phys. Rev. B: Condens. Matter Mater. Phys. 2013, 88, 35301 .

(48) Liu, X.; Xu, T.; Wu, X.; Zhang, Z.; Yu, J.; Qiu, H.; Hong, J.-H.; Jin, C.-H.; Li, J.-X.; Wang, X.-R.; et al. Top-down Fabrication of SubNanometre Semiconducting Nanoribbons Derived from Molybdenum Disulfide Sheets. Nat. Commun. 2013, 4, 1776.

(49) Wang, Y.; Feng, Y.; Chen, Y.; Mo, F.; Qian, G.; Yu, D.; Wang, Y.; Zhang, X. Morphological and Structural Evolution of WS 2 Nanosheets Irradiated with an Electron Beam. Phys. Chem. Chem. Phys. 2015, 17, 2678-2685.

(50) Wang, S.; Lee, G.-D.; Lee, S.; Yoon, E.; Warner, J. H. Detailed Atomic Reconstruction of Extended Line Defects in Monolayer MoS2. ACS Nano 2016, 10, 5419-5430.

(51) Zink, N.; Therese, H. A.; Pansiot, J.; Yella, A.; Banhart, F.; Tremel, W. In Situ Heating TEM Study of Onion-like WS2 and MoS2 Nanostructures Obtained via MOCVD. Chem. Mater. 2008, 20, 6571 .

(52) Hanzawa, A.; Shimada, T.; Hasegawa, T.; Sato, T.; Kamino, T.; Yonezawa, T. In-Situ TEM Observations of the Crystallization Process of Solution-Prepared MoS2 Amorphous Particles. J. Nanosci. Nanotechnol. 2009, 9, 6736-6740.

(53) José-Yacamán, M.; Lopez, H.; Santiago, P.; Galvan, D.; Garzon, I.; Reyes, A. Studies of MoS2 Structures Produced by Electron Irradiation. Appl. Phys. Lett. 1996, 69, 1065-1067.

(54) Kim, B. H.; Gu, H. H.; Yoon, Y. J. Atomic Rearrangement of a Sputtered MoS2 Film from Amorphous to a 2D Layered Structure by Electron Beam Irradiation. Sci. Rep. 2017, 7, 3874.

(55) Krivanek, O. L.; Chisholm, M. F.; Nicolosi, V.; Pennycook, T. J.; Corbin, G. J.; Dellby, N.; Murfitt, M. F.; Own, C. S.; Szilagyi, Z. S.; 
Oxley, M. P.; Pantelides, S. T.; Pennycook, S. J. Atom-by-Atom Structural and Chemical Analysis by Annular Dark-Field Electron Microscopy. Nature 2010, 464, 571-574.

(56) Nicolini, P.; Capozza, R.; Restuccia, P.; Polcar, T. Structural Ordering of Molybdenum Disulfide Studied via Reactive Molecular Dynamics Simulations. ACS Appl. Mater. Interfaces 2018, 10, 89378946.

(57) Vilá, R. A.; Rao, R.; Muratore, C.; Bianco, E.; Robinson, J. A.; Maruyama, B.; Glavin, N. R. In Situ Crystallization Kinetics of TwoDimensional MoS2. 2D Mater. 2018, 5, 11009.

(58) Lai, C.-C.; Meshkian, R.; Dahlqvist, M.; Lu, J.; Näslund, L.-Å.; Rivin, O.; Caspi, E.; Ozeri, O.; Hultman, L.; Eklund, P.; et al. Structural and Chemical Determination of the New Nanolaminated Carbide Mo 2 Ga 2 C from First Principles and Materials Analysis. Acta Mater. 2015, 99, 157-164.

(59) Sun, D.; Lu, W.; Le, D.; Ma, Q.; Aminpour, M.; Alcántara Ortigoza, M.; Bobek, S.; Mann, J.; Wyrick, J.; Rahman, T. S.; Bartels, L. An MoSx Structure with High Affinity for Adsorbate Interaction. Angew. Chem. 2012, 124, 10430-10434.

(60) Sutter, E.; Huang, Y.; Komsa, H.-P.; Ghorbani-Asl, M.; Krasheninnikov, A.; Sutter, P. Electron-Beam Induced Transformations of Layered Tin Dichalcogenides. Nano Lett. 2016, 16, $4410-4416$.

(61) Ma, Q.; Odenthal, P. M.; Mann, J.; Le, D.; Wang, C. S.; Zhu, Y.; Chen, T.; Sun, D.; Yamaguchi, K.; Tran, T.; et al. Controlled Argon Beam-Induced Desulfurization of Monolayer Molybdenum Disulfide. J. Phys.: Condens. Matter 2013, 25, 252201.

(62) Hong, J.; Hu, Z.; Probert, M.; Li, K.; Lv, D.; Yang, X.; Gu, L.; Mao, N.; Feng, Q.; Xie, L.; et al. Exploring Atomic Defects in Molybdenum Disulphide Monolayers. Nat. Commun. 2015, 6, 6293.

(63) Yu, Z. G.; Zhang, Y.-W.; Yakobson, B. I. An Anomalous Formation Pathway for Dislocation-Sulfur Vacancy Complexes in Polycrystalline Monolayer MoS2. Nano Lett. 2015, 15, 6855-6861.

(64) Hong, J.; Pan, Y.; Hu, Z.; Lv, D.; Jin, C.; Ji, W.; Yuan, J.; Zhang, Z. Direct Imaging of Kinetic Pathways of Atomic Diffusion in Monolayer Molybdenum Disulfide. Nano Lett. 2017, 17, 3383-3390.

(65) Li, Y.; Wang, H.; Xie, L.; Liang, Y.; Hong, G.; Dai, H. MoS2 Nanoparticles Grown on Graphene: An Advanced Catalyst for the Hydrogen Evolution Reaction. J. Am. Chem. Soc. 2011, 133, 72967299.

(66) Xie, X.; Makaryan, T.; Zhao, M.; Van Aken, K. L.; Gogotsi, Y.; Wang, G. MoS2 Nanosheets Vertically Aligned on Carbon Paper: A Freestanding Electrode for Highly Reversible Sodium-Ion Batteries. Adv. Energy Mater. 2016, 6, 1502161.

(67) Zhang, W.; Chuu, C.-P.; Huang, J.-K.; Chen, C.-H.; Tsai, M.-L.; Chang, Y.-H.; Liang, C.-T.; Chen, Y.-Z.; et al. Ultrahigh-Gain Photodetectors Based on Atomically Thin Graphene-MoS2 Heterostructures. Sci. Rep. 2015, 4, 3826.

(68) Gruber, E.; Wilhelm, R. A.; Pétuya, R.; Smejkal, V.; Kozubek, R.; Hierzenberger, A.; Bayer, B. C.; Aldazabal, I.; Kazansky, A. K.; Libisch, F.; Krasheninnikov, A. V.; Schleberger, M.; Facsko, S.; Borisov, A. G.; Arnau, A.; Aumayr, F. Ultrafast Electronic Response of Graphene to a Strong and Localized Electric Field. Nat. Commun. 2016, 7, 13948.

(69) Miwa, J. A.; Dendzik, M.; Grønborg, S. S.; Bianchi, M.; Lauritsen, J. V.; Hofmann, P.; Ulstrup, S. Van Der Waals Epitaxy of Two-Dimensional MoS2-graphene Heterostructures in Ultrahigh Vacuum. ACS Nano 2015, 9, 6502-6510.

(70) Shi, J.; Liu, M.; Wen, J.; Ren, X.; Zhou, X.; Ji, Q.; Ma, D.; Zhang, Y.; Jin, C.; Chen, H.; et al. All Chemical Vapor Deposition Synthesis and Intrinsic Bandgap Observation of MoS2/graphene Heterostructures. Adv. Mater. 2015, 27, 7086-7092.

(71) Liu, X.; Balla, I.; Bergeron, H.; Campbell, G. P.; Bedzyk, M. J.; Hersam, M. C. Rotationally Commensurate Growth of MoS2 on Epitaxial Graphene. ACS Nano 2016, 10, 1067-1075.

(72) Wan, W.; Li, X.; Li, X.; Xu, B.; Zhan, L.; Zhao, Z.; Zhang, P.; Wu, S.; Zhu, Z.; Huang, H.; et al. Interlayer Coupling of a Direct van Der Waals Epitaxial MoS 2/graphene Heterostructure. RSC Adv. 2016, 6, 323-330.
(73) Li, X.; Cai, W.; An, J.; Kim, S.; Nah, J.; Yang, D.; Piner, R.; Velamakanni, A.; Jung, I.; Tutuc, E.; Banerjee, S. K.; Colombo, L.; Ruoff, R. S. Large-Area Synthesis of High-Quality and Uniform Graphene Films on Copper Foils. Science 2009, 324, 1312-1314.

(74) Kidambi, P. R.; Bayer, B. C.; Blume, R.; Wang, Z.-J.; Baehtz, C.; Weatherup, R. S.; Willinger, M.-G.; Schloegl, R.; Hofmann, S. Observing Graphene Grow: Catalyst-Graphene Interactions during Scalable Graphene Growth on Polycrystalline Copper. Nano Lett. 2013, 13, 4769-4778.

(75) Kong, D.; Wang, H.; Cha, J. J.; Pasta, M.; Koski, K. J.; Yao, J.; Cui, Y. Synthesis of MoS2 and MoSe2 Films with Vertically Aligned Layers. Nano Lett. 2013, 13, 1341-1347.

(76) Fei, L.; Ng, S. M.; Lu, W.; Xu, M.; Shu, L.; Zhang, W.-B.; Yong, Z.; Sun, T.; Lam, C. H.; Leung, C. W.; et al. Atomic-Scale Mechanism on Nucleation and Growth of Mo2C Nanoparticles Revealed by in Situ Transmission Electron Microscopy. Nano Lett. 2016, 16, 78757881.

(77) Moser, J.; Levy, F. Growth Mechanisms and near-Interface Structure in Relation to Orientation of MoS 2 Sputtered Thin Films. J. Mater. Res. 1992, 7, 734-740. 